\title{
Differences in conformational dynamics within the Hsp90 chaperone family reveal mechanistic insights
}

\author{
Christian Graf ${ }^{\dagger}$, Chung-Tien Lee ${ }^{\dagger}$, L. Eva Meier-Andrejszki, Minh T. N. Nguyen and Matthias P. Mayer*
}

Ruprecht-Karls-Universität Heidelberg, Zentrum für Molekulare Biologie der Universität Heidelberg, Heidelberg, Germany

Edited by:

Walid A. Houry, University of

Toronto, Canada

Reviewed by:

Brian Christopher Freeman,

University of Illinois,

Urbana-Champaign, USA

Chrisostomos Prodromou,

University of Sussex, UK

Jason C. Young, McGill University,

Canada

*Correspondence:

Matthias P. Mayer,

Ruprecht-Karls-Universität

Heidelberg, Zentrum für Molekulare

Biologie der Universität Heidelberg,

DKFZ-ZMBH-Alliance,

Ruprecht-Karls-Universität

Heidelberg, Im Neuenheimer Feld

282, 69120 Heidelberg, Germany

e-mail:m.mayer@zmbh.

uni-heidelberg.de

${ }^{\dagger}$ Present address:

Christian Graf, Novartis Pharma AG,

Basel, Switzerland;

Chung-Tien Lee, Max-Planck

Institute for Biophysical Chemistry,

Göttingen, Germany
The molecular chaperones of the Hsp90 family are essential in all eukaryotic cells. They assist late folding steps and maturation of many different proteins, called clients, that are not related in sequence or structure. Hsp90 interaction with its clients appears to be coupled to a series of conformational changes. Using hydrogen exchange mass spectrometry (HX-MS) we investigated the structural dynamics of human Hsp90 $\beta$ (hHsp90) and yeast Hsp82 (yHsp82). We found that eukaryotic Hsp90s are much more flexible than the previously studied Escherichia coli homolog (EcHtpG) and that nucleotides induce much smaller changes. More stable conformations in yHsp82 are obtained in presence of co-chaperones. The tetratricopeptide repeat (TPR) domain protein Cpr6 causes a different amide proton protection pattern in yHsp82 than the previously studied TPR-domain protein Sti1. In the simultaneous presence of Sti1 and Cpr6, protection levels are observed that are intermediate between the Sti1 and the Cpr6 induced changes. Surprisingly, no bimodal distributions of the isotope peaks are detected, suggesting that both co-chaperones affect both protomers of the Hsp90 dimer in a similar way. The cochaperones Sba1 was found previously in the crystal structure bound to the ATP hydrolysis-competent conformation of Hsp90, which did not allow to distinguish the mode of Sba1-mediated inhibition of Hsp90's ATPase activity by stabilizing the pre- or posthydrolysis step. Our HX-MS experiments now show that Sba1 binding leads to a protection of the ATP binding lid, suggesting that it inhibits Hsp90's ATPase activity by slowing down product release. This hypothesis was verified by a single-turnover ATPase assay. Together, our data suggest that there are much smaller energy barriers between conformational states in eukaryotic Hsp90s than in EcHtpG and that co-chaperones are necessary in addition to nucleotides to stabilize defined conformational states.

Keywords: hydrogen exchange mass spectrometry, chaperones, Hsp90, dynamics, conformation

\section{INTRODUCTION}

Hsp90 chaperones control stability and activity of some 200 native or near native protein clients, most of which are regulatory components of signal transduction pathways such as receptors, protein kinases and transcription factors (Pratt and Toft, 1997; Pearl and Prodromou, 2000; Young et al., 2001; Wegele et al., 2004; DeZwaan and Freeman, 2008).Through chaperoning these clients Hsp90s are involved in the regulation of cell homoeostasis, cell cycle, proliferation, differentiation, and programmed cell death.

The Hsp90 family of proteins is evolutionary highly conserved with $60 \%$ sequence identity between yeast and human and $40 \%$ between E. coli and human members (Figure 1). In yeast and human two cytosolic Hsp90 proteins with 97 and $86 \%$ sequence identity are found, yeast $\mathrm{Hsc} 82$ and yeast Hsp82 and human Hsp90 $\alpha$ and human Hsp90ß, that are expressed at different level and in cell type-specific manner, respectively (Ghaemmaghami et al., 2003; Sreedhar et al., 2004). The homodimeric Hsp90 proteins consist of an $\mathrm{N}$-terminal nucleotide-binding domain (NBD), a middle domain (MD) and a C-terminal dimerization domain (DD) (Figure 2A). Crystal structures of full-length constructs of yeast Hsp82, of E. coli HtpG, and of the canine Grp94 show similar domain structures but very different domain orientations, suggesting significant conformational dynamics (Ali et al., 2006; Shiau et al., 2006; Dollins et al., 2007). Based on the structurally related GHKL-ATPases DNA gyrase B and MutL the ATPase cycle of Hsp90 was proposed to involve several conformational changes including ATP-dependent docking of NBD and MD of the same monomer and a dimerization of the two NBDs in the Hsp90 dimer resulting in a twisted intertwined structure (Ali et al., 2006; Pearl and Prodromou, 2006). Several studies using electron microscopy, small angle X-ray scattering (SAXS) and fluorescence resonance energy transfer (FRET) confirmed domain movements in Hsp90 proteins (Shiau et al., 2006; Southworth and Agard, 2008; Hessling et al., 2009; Krukenberg et al., 2009; Mickler et al., 2009). However, it is unclear, whether there is a uniform cycle of conformational changes or whether Hsp90 proteins differ in the basis for their chaperone activity.

A method well suitable to analyze these fundamental concepts of the chaperone mechanism is hydrogen- ${ }^{1} \mathrm{H} /{ }^{2} \mathrm{H}$-exchange (HX) mass spectrometry (MS) because it enables insights into global and local protein stability, secondary structure dynamics and 


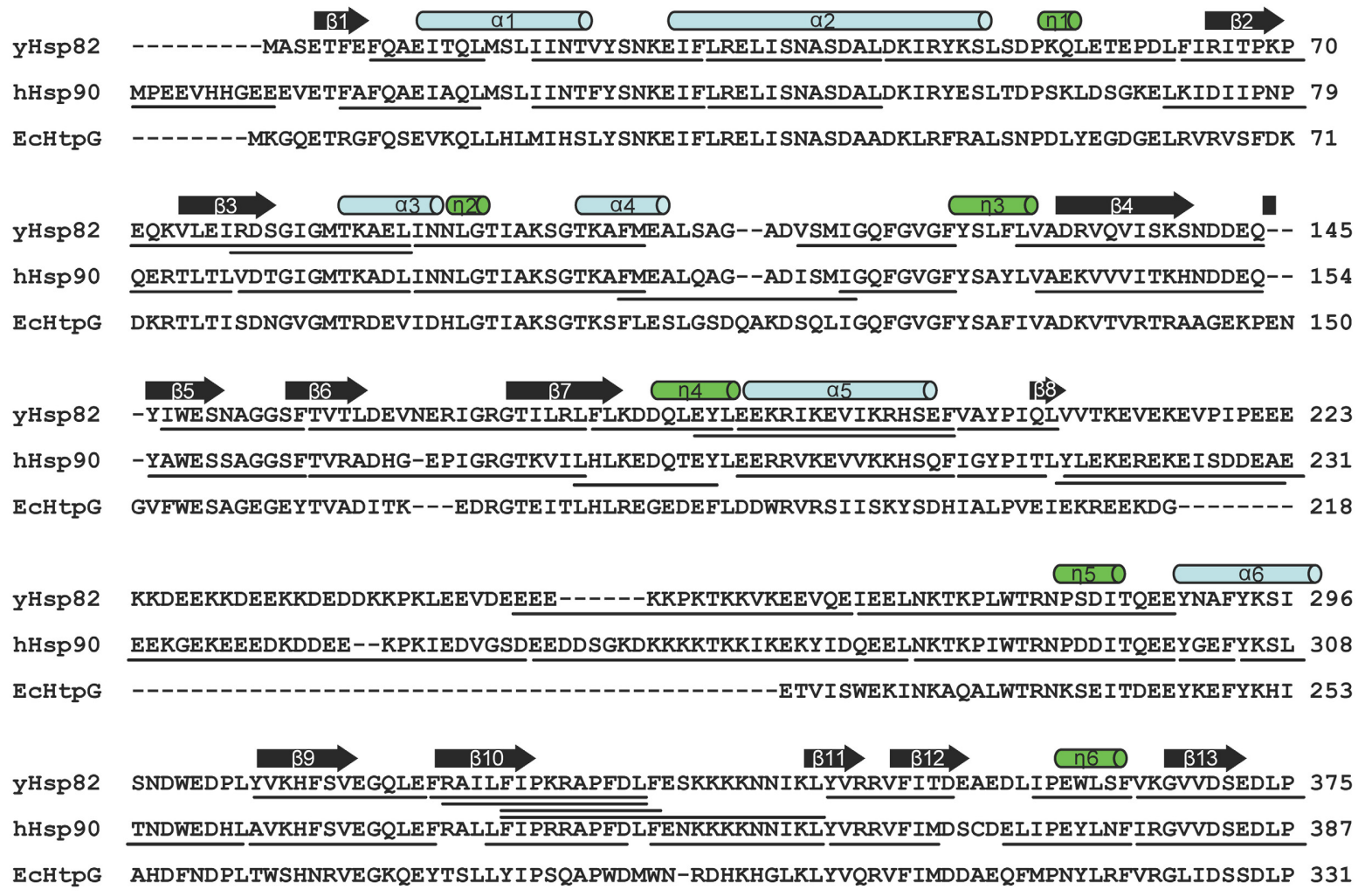

YHsp82 LNLSREMLQQNKIMKVIRKNIVKKLIEAFNE IAED-SEQFEKFYSAFSKNIKLGVHEDTQNRAALAKLLRYNSTK--SV

hHsp90 LNISREMLQQSKILKVIRKNIVKKCLELFSELAED-KENYKKFYEAFSKNLKLGIHEDSTNRRRLSELLRYHTSQ--SG 463

EcHtpG LNVSREILQDSTVTRNLRNALTKRVLQMLEKLAKDDAEKYQTFWQQFGLVLKEGPAEDFANQEAIAKLLRFASTHTDSS 410

YHsP82 DELTSLTDYVTRMPEHQKNIYYITGESLKAVEKSPFLDALKAKNEEVLFLTDPIDEYAFTQLKEFEGKTLVDITKD-FE 528

hHsP90 DEMTSLSEYVSRMKETQKSIYYITGESKEQVANSAFVERVRKRGFEVVYMTEPIDEYCVQQLKEFDGKSLVSVTKEGLE 542

EcHtpG AQTVSLEDYVSRMKEGQEKIYYITADSYAAAKSSPHLELLRKKGIEVLLLSDRIDEWMMNYLTEFDGKPFQSVSKVDES 489

YHSP82 LEETDEEKAE-REKEIKEYEPLTKALKEILGDQVEKVVVSYKLIDAPAAIRTGQFGWSANMERIMKAQALRDSSMSSYM

hHsp90 LPEDEEEKKK-MEESKAKFENLCKLMKEILDKKVEKVTISNRLVSSPCCIVTSTYGWTANMERIMKAQALRDNSTMGYM 620

EcHtPG LEKLADEVDESAKEAEKALTPFIDRVKALLGERVKDVRLTHRLTDTPAIVSTDADEMSTQMAKLFAAAGQKVP------ 562

YHsp82 SSKKTFEISPKSPIIKELKKRVDEGGAQDKTVKDLTKLLYETALLTSGFSLDEPTSFASRINRLISLGLNIDEDEETET 686

hHSP90 MAKKHLEINPDHPIVETLRQKAEAD-KNDKAVKDLVVLLFETALLSSGFSLEDPQTHSNRIYRMIKLGLGIDEDEVAAE 698

EcHtPG EVKYIFELNPDHVLVKRAADTEDEA-----KFSEWVELLLDQALLAERGTLEDPNLFIRRMNQLLVS-1

$\begin{array}{lll}\text { yHsp82 } & \text { APEASTAAPVEEVPAD---TEMEEVD } & 709 \\ \text { hHsp90 } & \text { EPNAAVPDEIPPLEGDEDASRMEEVD } & 724 \\ \text { EcHtpG } & ---0 & \end{array}$

FIGURE 1 | Comparison of human, yeast and E. coli Hsp90 proteins. 


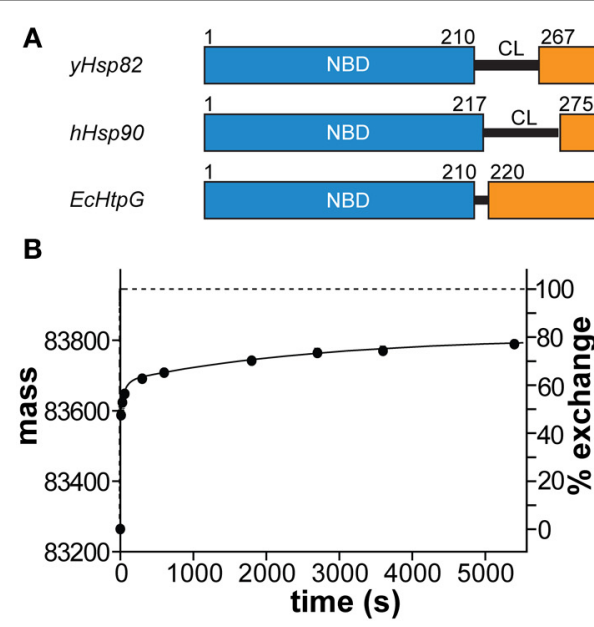

FIGURE 2 | Comparison of human, yeast and E. coli Hsp90 proteins. (A) Domain structure of human $\mathrm{Hsp} 90 \beta$ (hHsp90), S. cerevisiae Hsp82 (yHsp82) and E. coli HtpG (EcHtpG) proteins. NBD, nucleotide binding domain; $C L$, charged linker; $M D$, middle domain; $D D$, dimerization domain. (B) $\mathrm{HX}$ kinetics of full-length hHsp90. The mass of hHsp90 and the percentage hydrogen exchange, respectively, is plotted vs. the incubation time in $\mathrm{D}_{2} \mathrm{O}$. The data points are averages of up to four independent experiments and the error bars represent the standard error of mean. The solid line is a fit of a tri-exponential equation $\left[Y=Y_{1} \cdot\left(1-\exp \left(-k_{1} \cdot t\right)\right)+\right.$ $\left.Y_{2} \cdot\left(1-\exp \left(-k_{2} \cdot t\right)\right)+Y_{3} \cdot\left(1-\exp \left(-k_{3} \cdot t\right)\right)\right]$ to the data points. The dashed line represents the theoretical exchange curve including the
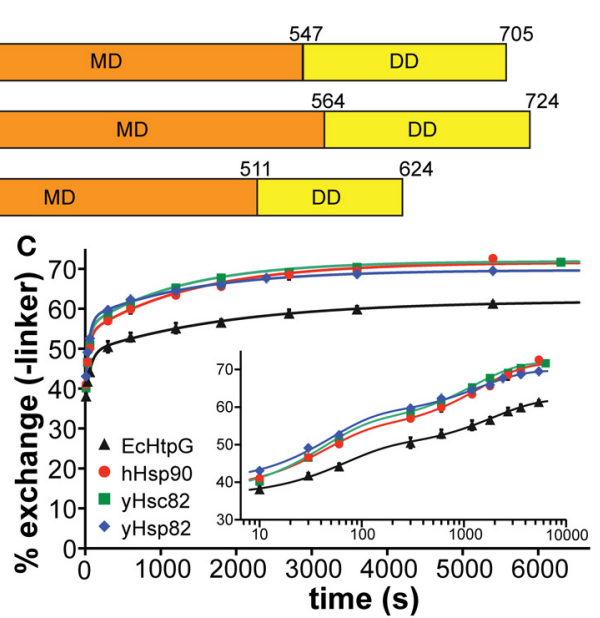

back-exchange during the desalting process calculated for the completely unfolded Hsp90 protein using the HXPep program (courtesy of Z. Zhang) (C) HX kinetics of full-length hHsp90 (black circles), yHsc82 (green squares), yHsp82 (blue diamonds), and EcHtpG (black triangles; data from (Graf et al., 2009) for comparison). Relative exchange minus the amide hydrogens of the linker $\left[\left(\mathrm{H}_{\mathrm{t}}-\mathrm{H}_{\text {linker }}\right) /\left(\mathrm{H}_{\text {total }}-\mathrm{H}_{\text {linker }}\right)\right]$ is plotted vs. the time in $\mathrm{D}_{2} \mathrm{O}$. The data points are averages of two to four independent measurements. The error bars represent standard error of mean. The solid lines are the fits of a bi-exponential equation $\left[Y=Y_{1} \cdot\left(1-\exp \left(-k_{1} \cdot t\right)\right)\right.$ $\left.+Y_{2} \cdot\left(1-\exp \left(-k_{2} \cdot t\right)\right)+Y_{3}\right]$ to the data. The inset shows the same data with a logarithmic time scale. induced alterations in protein conformation (Hoofnagle et al., 2003; Wales and Engen, 2006). We recently analyzed the conformational dynamics of E. coli $\mathrm{HtpG}$ (EcHtpG) (Graf et al., 2009). We found that HtpG is rather dynamic in the absence of nucleotides and in the presence of ADP. ATP induced a relatively slow stepwise transition to a more tightly folded state, which we called the tensed-state in contrast to the nucleotidefree relaxed state. Here we analyzed three eukaryotic homologs of HtpG, human Hsp90ß (hHsp90), yeast $\mathrm{Hsc} 82$ (yHsc82), and yeast Hsp82 (yHsp82) to investigate whether the conformational changes detected for HtpG are conserved throughout evolution. We found that yeast and human Hsp90s differ significantly from EcHtpG in respect to rigidity and conformational dynamics and how nucleotides influence conformational dynamics.

\section{RESULTS}

\section{DEUTERON INCORPORATION INTO HUMAN AND YEAST Hsp90s}

To analyze the conformational dynamics of human and yeast Hsp90s we performed HX-MS experiments essentially as described earlier (Rist et al., 2003, 2006; Graf et al., 2009). We found that the yeast and human Hsp90 proteins are extremely flexible at the secondary structure level exchanging in the nucleotide-free state close to $50 \%$ of their amide hydrogens within $10 \mathrm{~s}$ and almost $80 \%$ within $1 \mathrm{~h}$ (Figures 2B,C). The exchange kinetics of the eukaryotic Hsp90s contrast the earlier investigated EcHtpG (Graf et al., 2009), which was more stable exchanging only $38 \%$ of its amide hydrogens within $10 \mathrm{~s}$ and around $60 \%$ within $1 \mathrm{~h}$. This difference in mobility is not related to the different length of the charged linker connecting NBD and MD (see
Figure 2A) and also apparent when the numbers of linker amide hydrogens are subtracted from the data (Figure 2C). The difference was also not due to the physiological role of the Hsp90 as constitutively high abundant housekeeping or heat shock protein, since we did not detect significant differences between $\mathrm{yHsc} 82$ and yHsp82 at this level.

Online peptic digestion under quench conditions at $0^{\circ} \mathrm{C}$ allowed us to map fast and slow exchanging regions in the proteins with a sequence coverage of 82,65 , and $69 \%$ for the hHsp90, yHsc82 and yHsp82, respectively, including parts of the charged linker, which is not observed in any crystal structure (Figure 1). High secondary structure stability was observed in the NBD except for the ATP-lid [residues 99-133 in hHsp90; homologous to the ATP-lid in GHKL-ATPases (Prodromou et al., 1997a)]. MD and DD are much more flexible than the NBD (Figure 3). Not surprisingly, the charged linker (residues 216-275 in hHsp90), which connects NBD and MD and which is symbolized as dashed line in Figure 3, exchanged almost all of its amide hydrogen within $10 \mathrm{~s}$. A high degree of dynamics was observed in the catalytic loop in the MD (residues 382-402 in hHsp90) and in three structural elements implicated in substrate binding: the loop, which contains tryptophan 312 in hHsp90 [position 300 in yHsp82; (Sato et al., 2000)], the so-called Src-loop [residues 324340 in yHsp82 and not resolved in the structure; (Meyer et al., 2003)], and $\alpha$ helix 15 (helix 21 in EcHtpG (Harris et al., 2004); Figure 3). Even one or both of the helices involved in the dimer interface incorporated deuterons to a high degree within $10 \mathrm{~min}$ consistent with a fast dimer-monomer equilibrium and frequent opening of the DD (Figure 3 right panels) (Richter et al., 2001; 


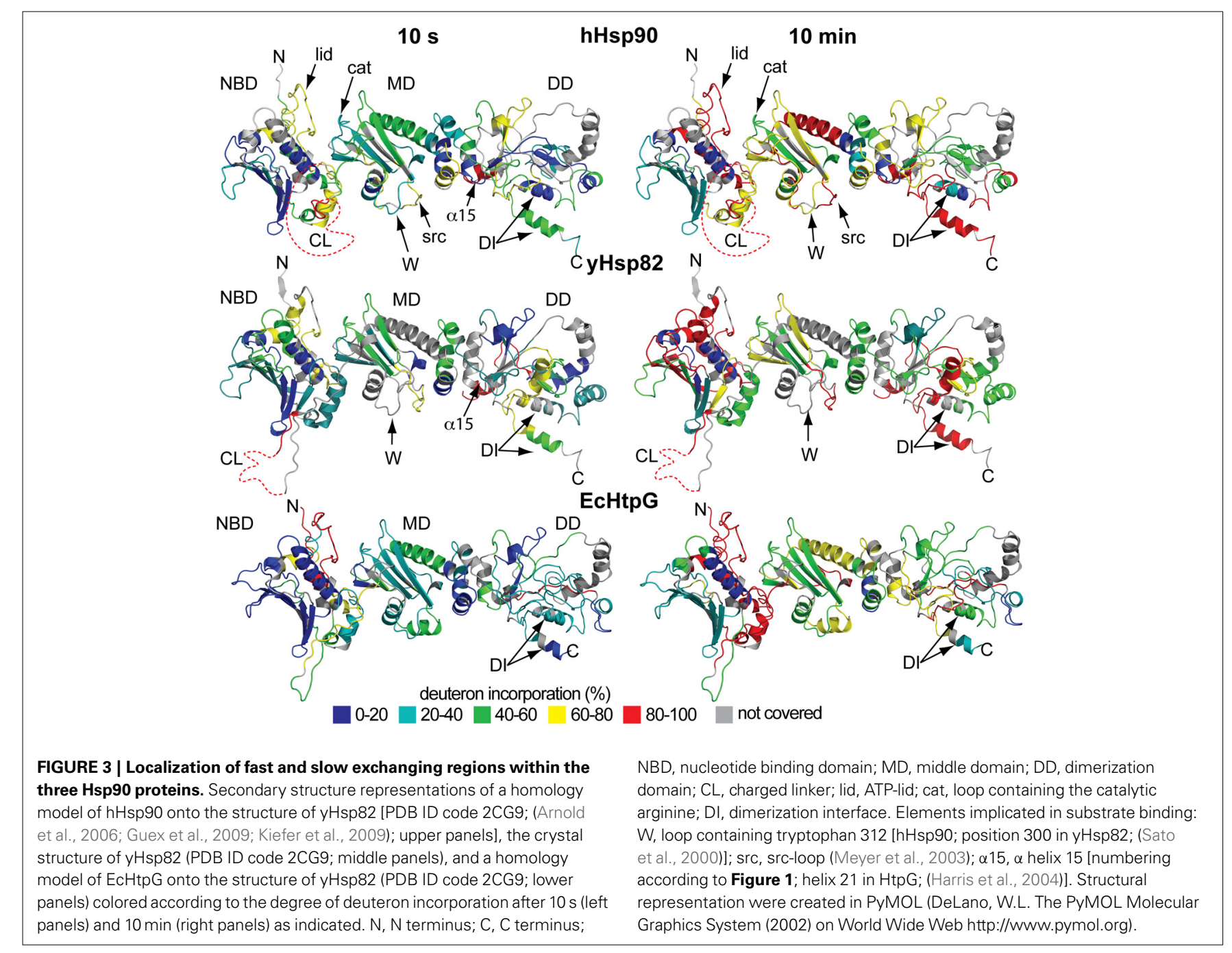

Ratzke et al., 2010). Deuteron incorporation in yHsc82 was very similar to $\mathrm{yHsp} 82$, indicating that the heat-inducible isoform is not necessarily more stable.

The difference in conformational dynamics between the eukaryotic Hsp90 proteins and EcHtpG were found in many parts of MD and DD. In particular, one of the helices involved in the dimer interface is more stable in EcHtpG than its counterpart in yeast or human Hsp90 suggesting a less dynamic dimer-monomer equilibrium and/or opening of the DD.

\section{EFFECTS OF NUCLEOTIDES ON HYDROGEN EXCHANGE IN Hsp90 PROTEINS}

To analyze the influence of nucleotides on the conformational dynamics we pre-incubated the three Hsp90 proteins with a large excess of ADP, ATP, and the non-hydrolyzable ATP analog AMPPNP for at least $10 \mathrm{~min}$ before the start of the proton/deuteron exchange reaction. As compared to the previously analyzed EcHtpG, nucleotides only induced small changes in conformational dynamics of the eukaryotic Hsp90 proteins (Figure 4). For hHsp90 we found that all three nucleotides led to a small protection at short time intervals $(10,14$, and 23 hydrogens for ADP, ATP and AMPPNP at $10 \mathrm{~s}$ ) (Figure 4A). For yHsp82 only in the presence of AMPPNP a significant protection was observed in the full-length protein (on average 18 protons) (Figure 4A). These observations are in striking contrast to the 50 protons protection observed in EcHtpG upon addition of ATP (Graf et al., 2009), indicating that in the eukaryotic proteins ATP does not induce a similarly rigid state comparable to the "tensed state" of EcHtpG.

Mapping the nucleotide-induced differences onto the sequence of hHsp90 revealed that a stabilizing effect of ATP was observed in the ATP-lid (residues 99-114 and 126-133; Figure 4C, left panel, black bars). A small stabilization was also observed throughout the entire protein down to the very C-terminus. In particular, the segment following the catalytic arginine 392 exhibited a significant stabilization in the presence of ATP suggesting a contact between the NBD and the MD upon ATP binding consistent with the currently favored ATPase mechanism for Hsp90 proteins. In the presence of ADP, the situation is quite different for hHsp90. Though a protection of the ATP-lid was also observed in the presence of ADP, several segments of significant deprotection occurred throughout 

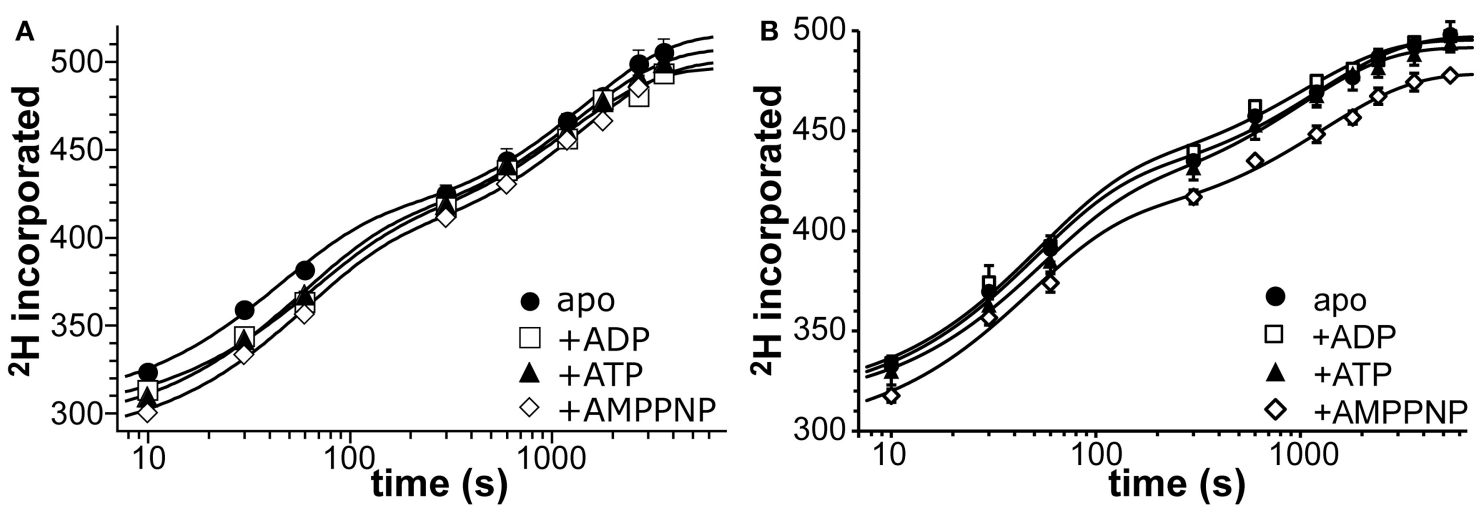

C hHsp90
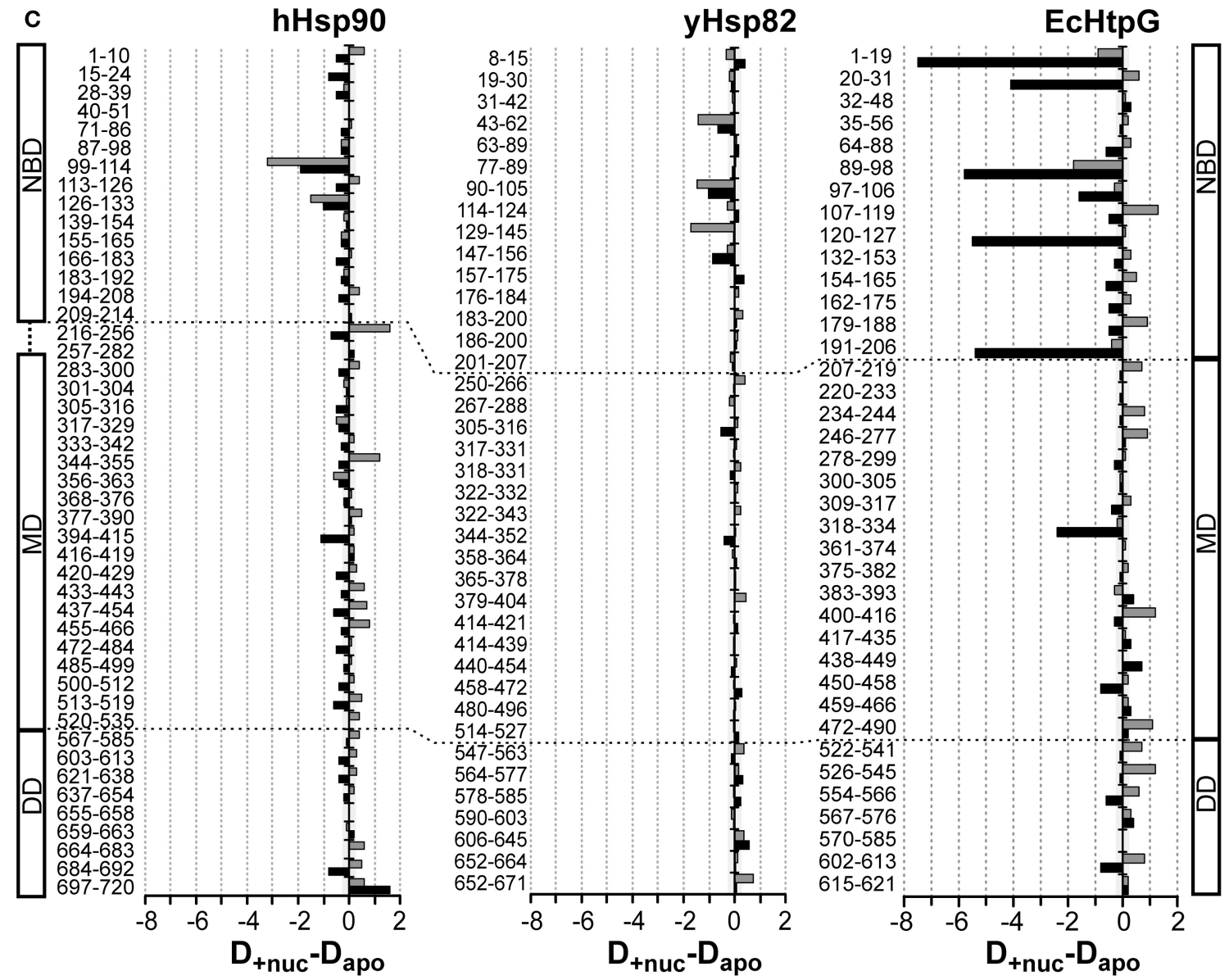

FIGURE 4 | Nucleotide dependent changes in the HX kinetics of Hsp90 proteins. Deuteron incorporation into full-length $\mathrm{hHsp90}$ (A) and yHsp82 (B) in the absence of nucleotides (black circles) and in the presence of ADP (open squares), ATP (black triangles), and AMPPNP (open diamonds). The solid lines are fits of a bi-exponential equation to the data. (C) Difference plots of deuterons incorporated in the presence of ATP (black bars) and ADP (gray bars) minus deuterons incorporated in the absence of nucleotides into hHsp90 (left panel), yHsp82 (middle panel) and EcHtpG (right panel, data from Graf et al., 2009) after $30 \mathrm{~s}$ in $D_{2} \mathrm{O}$. The numbers to the left of each panel indicate the corresponding peptic peptide. Presented are the means of three independent experiments. Domain structure of $\mathrm{hHsp} 90$ and $\mathrm{EcHtpG}$ are shown left and right of the graph. Dashed lines indicate domain boundaries in the three proteins. the protein contrasting the protection in the presence of ATP (Figure 4C left panel, gray bars). Part of the charged linker (residues 216-256), the src-loop (residues 344-355), and the helical coiled-coil of the MD (residues 433-466) exhibited this deprotection.
For yHsp82 we observed in the presence of ATP a small protection in the NBD and two segments of the MD and a deprotection in the DD (Figure 4C, middle panel, black bars). In the presence of ADP, protection was observed in the NBD and deprotection in parts of the MD and the DD. In particular, the segment 
encompassing the dimerization interface was deprotected in the presence of ADP.

It was shown previously the AMPPNP leads to a more stable $\mathrm{N}$-terminal dimerization in yHsp82 (Prodromou et al., 2000). We therefore repeated the experiment with yHsp82 in the presence AMPPNP (Figure 5). The observed protection in the N-terminal domain was more prominent as in the presence of ATP or ADP but again not comparable to the extent observed in EcHtpG. Interestingly, we also observed a significant protection in the $\mathrm{MD}$, segment 480-496, which forms the interface to the DD, and in the dimerization interface. The protection in segment 480-496 suggests less movements of the MD relative to the $\mathrm{DD}$, consistent with a more stable $\mathrm{N}$-terminal dimerization. The protection in the dimer interface suggests a decrease of protomer dissociation of the Hsp90 dimer.

Taken together, significant nucleotide-dependent changes of the conformational dynamics were observed in the eukaryotic

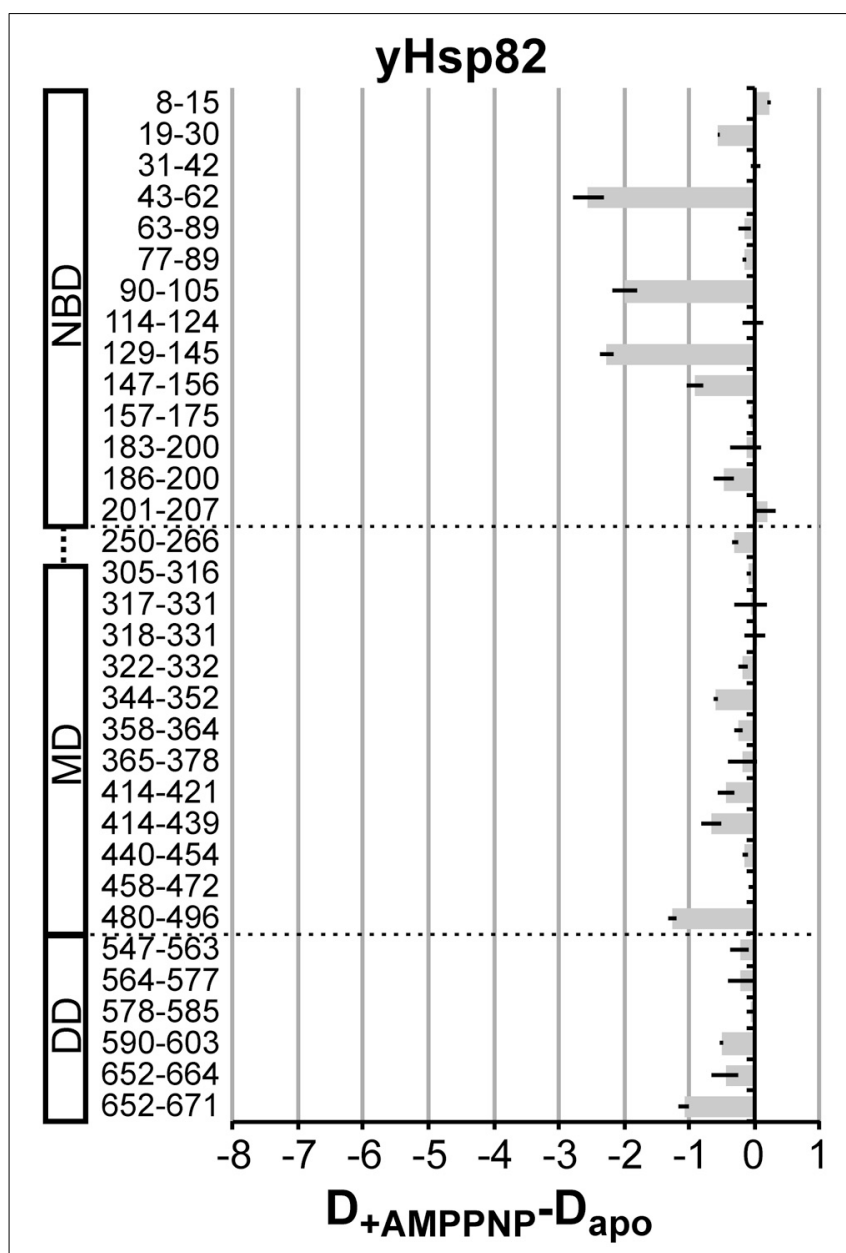

FIGURE 5 | AMPPNP-induced changes in the HX kinetics in yHsp82. Difference plots of deuterons incorporated in the presence of AMPPNP minus deuterons incorporated in the absence of nucleotides into yHsp82 after $30 \mathrm{~s}$ in $\mathrm{D}_{2} \mathrm{O}$. The numbers to the left indicate the corresponding peptic peptide. Presented are the means of three independent experiments. Domain structure of $\mathrm{yHsp90}$ is shown left of the graph. Dashed lines indicate domain boundaries.
Hsp90s not only in the NBD but also in MD and DD, but the extent of protection was smaller than in the previously studied EcHtpG and not observed in all the same regions (see Figure 4C right panel for comparison, reproduced from (Graf et al., 2009); please, note the different scales).

\section{SUBUNIT EXCHANGE IN Hsp90 PROTEINS}

Our HX-MS experiments demonstrated that Hsp90 proteins exhibit striking differences in dynamics of the C-terminal helices, which are involved in the dimerization interface. These findings suggest specific differences in the dimermonomer equilibrium and/or C-terminal opening. Therefore, we measured the subunit exchange rates using a Förster resonance energy transfer (FRET) assay as described by (Hessling et al., 2009) by labeling single cysteine variants of EcHtpG (HtpG-E58C), yHsp82 (Hsp82-E57C) and hHsp90 (Hsp90ß-E20C,C366A,C412T,C521A,C564T,C589A,C590A) with ATTO488 and ATTO550. Consistent with our HX-MS data, subunit exchange rates for human and yeast Hsp90 were about 5 and 10-fold higher than for EcHtpG (Figure 6 and Table 1). ATP and ADP had no significant influence on the exchange rates for yHsp82 and EcHtpG. AMPPNP drastically reduced subunit exchange rates for both Hsp90 proteins as had been shown before for yHsp82 (Hessling et al., 2009). For human Hsp90, ATP reduced the subunit exchange rates by $30 \%$, while ADP had no influence on the exchange rates. These data are consistent with the differences in protection observed in the segments covering the dimerization interface in EcHtpG, yHsp82 and hHsp90.

\section{CHANGES IN CONFORMATIONAL DYNAMICS OF yHsp82 UPON BINDING OF CPr6}

Since the eukaryotic Hsp90s are regulated by a number of cochaperones, which are absent in E. coli, we hypothesized that the

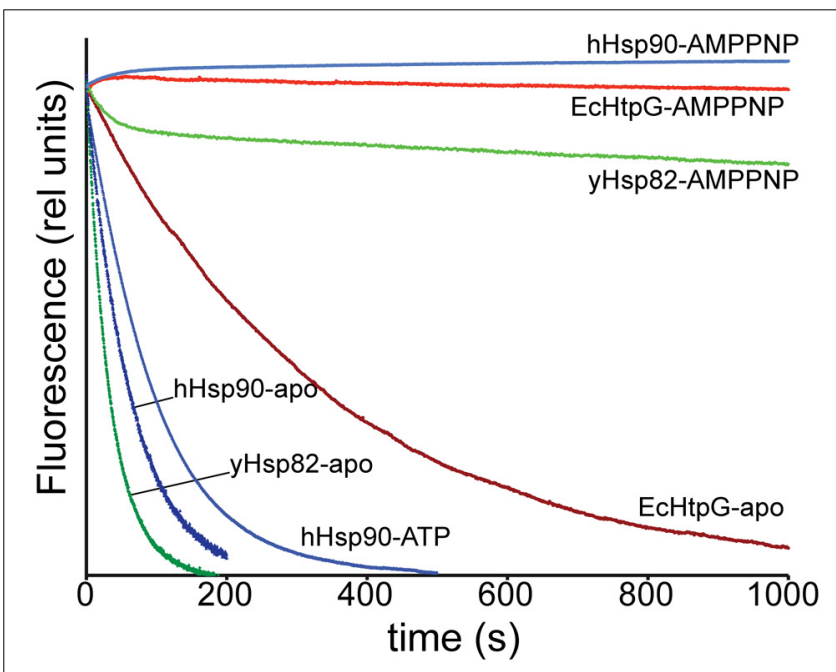

FIGURE 6 | Subunit-exchange rates differ significantly in Hsp90 proteins. Complexes of ATTO 488 and ATTO 550 labeled single cysteine variants of $\mathrm{EcHtpG}$ (red), yHsp82 (green) and $\mathrm{hHsp90}$ (blue) were rapidly mixed with a 10-fold excess of unlabeled protein in the absence of nucleotide (dark colors) or the presence of ATP or AMPPNP as indicated. 
Table 1 | Subunit exchange rates of Hsp90 proteins.

\begin{tabular}{llllc}
\hline Exchange rate $\left(\mathbf{s}^{-1}\right)$ & apo & +ATP & +ADP & +AMPPNP \\
\hline HtpG & 0.0028 & 0.0035 & 0.0036 & $\approx 2.8 \cdot 10^{-5}$ \\
Hsp82 & 0.030 & 0.027 & 0.030 & $0.034 / 4.8 \cdot 10^{-4}$ \\
Hsp90 $\beta$ & 0.016 & 0.010 & 0.016 & 0 \\
\hline
\end{tabular}

eukaryotic proteins may need the help of co-chaperones to arrive at the same tensed state as EcHtpG. To address this hypothesis, we analyzed the conformational changes of yHsp82 in the presence of the co-chaperones Cpr6, Stil and Sba1.

Cpr6 is a yeast member of the family of large peptidylprolyl-cis/trans-isomerases, which interacts with the C-terminal MEEVD-motif of Hsp90 through its tetratricopeptide repeat (TPR) domain. When performing HX-MS experiments with yHsp82 in the presence of Cpr6 we could not observe the interaction of Cpr6's TPR-domain and yHsp82's MEEVD-motif directly, because the C-terminal 38 residues were not covered by peptic peptides that we could detect consistently in HX-MS experiments. However, we detected significant protection in all three domains of yHsp82 (Figures 7A,D). Most prominent was the protection in helix $\alpha 18$ (aa 665-671), which is involved in the dimer interface, suggesting that Cpr6 stabilizes C-terminal dimerization. Protection was also observed in segment 480-496, which is situated at the hinge between $\mathrm{MD}$ and $\mathrm{DD}$, suggesting reduced relative rotational movement between $\mathrm{MD}$ and DD. Some protection was also observed in the NBD notably in the $\beta$-sheet (aa 129-156), in the ATP lid (aa 90-105) and in helix $\alpha 5$ (aa 183-200), which is at the interface between NBD and MD in the crystal structure of the closed conformation of yHsp82 (Ali et al., 2006), suggesting that the relative movement of NBD and MD is also reduced in the presence of Cpr6. Of note, only a small protection was observed in regions in the NBD (aa 8-30) that interact with each other during N-terminal dimerization, suggesting that Cpr6 does not significantly stabilize the $\mathrm{N}$-terminally dimerized state of Hsp90.

This exchange pattern of yHsp82 in the presence of Cpr6 has similarities but also striking differences to the pattern previously observed for $\mathrm{yHsp} 82$ in the presence of Stil (see Figure 7C, data from Lee et al., 2012 for comparison), which becomes more obvious when presented as difference plot (Figure 8A, left panel and Figure 8B, heat map). Positive values indicate regions that are more protected in the presence of Stil than in the presence of Cpr6, negative values are more protected in the presence of Cpr6 than in the presence of Stil. In the presence of Stil segments 43-62, 114-124, and 318-331 were more protected than in the presence of Cpr6. In contrast, region 129-156 was more protected in the presence of Cpr6 than in the presence of Stil.

Kinetic data demonstrated that Cpr6 can bind at the same time as Stil and that Cpr6 promotes dissociation of Stil in the function cycle of Hsp90 (Li et al., 2010). To understand the molecular basis of this observation we determined deuteron incorporation in the presence of stoichiometric concentrations of Cpr6 and Stil (yHsp82:Cpr6:Stil = 2:1:1; Figure 7B). Overall, the observed pattern is more similar to the pattern induced by Cpr6 than the Stil-induced pattern
(Figures 8A,C,D). Notably, the Stil-induced protection in regions 43-62 and 114-124 are strongly reduced as compared to the situation in the presence of Stil. In two regions (aa 250-266, 344-352) protection was observed when both co-chaperones were present, while no significant protection could be detected in the presence of either co-chaperone alone.

Taken together, our data demonstrate that Cpr6 and Sti1 induce different protection pattern in $\mathrm{yH} p 82$ indicating that they stabilize yHsp82 in defined distinguishable conformations.

\section{CHANGES IN CONFORMATIONAL DYNAMICS OF yHsp82 UPON BINDING OF Sba1}

The co-chaperone Sba1/p23 is known to form a complex with Hsp90 in the presence of AMPPNP (Johnson and Toft, 1995; Sullivan et al., 1997; Fang et al., 1998; Prodromou et al., 2000; Richter et al., 2004; Siligardi et al., 2004). We measured HX in yHsp82 in the presence of Sbal and AMPPNP and compared the results with HX in yHsp82 in the presence of AMPPNP. The difference plot in Figure 9A shows that a protection is observed in the entire protein. The crystal structure of yHsp82 $\Delta(221-255)-$ A107N variant in complex with Sbal and AMPPNP revealed residues 12-21, 27, 108-113 (ATP lid), 151-155, 315, 375, 387, 388 , and 391 of Hsp82 as major interaction sites for Sbal (Figure 9B). For most part our data are consistent with these interaction sites since we observed a significant protection in segments that contain the respective residues (segments 8-15, 19-30, 147-156, 365-378) or that are immediately adjacent to the interacting residues (90-105, 114-124) (Figures 9A,B). In addition, we found many segments in all three domains that exhibit strong protection in the presence of Sbal but that do not have any direct contact sites. In particular, strong protection was observed in residues 365-378, which in the crystal structure (Ali et al., 2006) make polar and hydrophobic contacts to residues 114-120 in the NBD; in the dimer interface (segment 652-671), indicating reduced dissociation of the $\mathrm{yHsp} 82$ dimer and/or C-terminal opening; and in the hinge region between MD and DD (segment 480-496), suggesting reduced movement of the MD relative to the DD. These data are consistent with the stabilization of yHsp82 in the closed conformation as suggested by biochemical experiments and revealed in the crystal structure (Richter et al., 2004; Ali et al., 2006). Our data demonstrate that Sbal reduces the conformational dynamics of the ATP lid as indicated by the protection in residues 90-105 and 114-124, which is consistent with mutant analysis (Siligardi et al., 2004). This suggests that Sbal inhibits ATP hydrolysis by preventing product release. To test this hypothesis we performed single-turnover ATPase assays in the absence and presence of Sba1, since product release is not relevant in this assay. As shown in Figure 9C Sbal did not inhibit significantly the ATPase activity of yHsp82 under single turnover conditions, which contrasts the observed inhibition under steady state conditions, demonstrating that Sbal inhibits product release. Thus, Sba1 prolongs the life-time of the closed conformation of Hsp90 as previously proposed (Ali et al., 2006; Prodromou, 2012) and consistent with Sbal's proposed ability to stabilize Hsp90 client complexes (Richter et al., 2004; Siligardi et al., 2004; McLaughlin et al., 2006). 
A

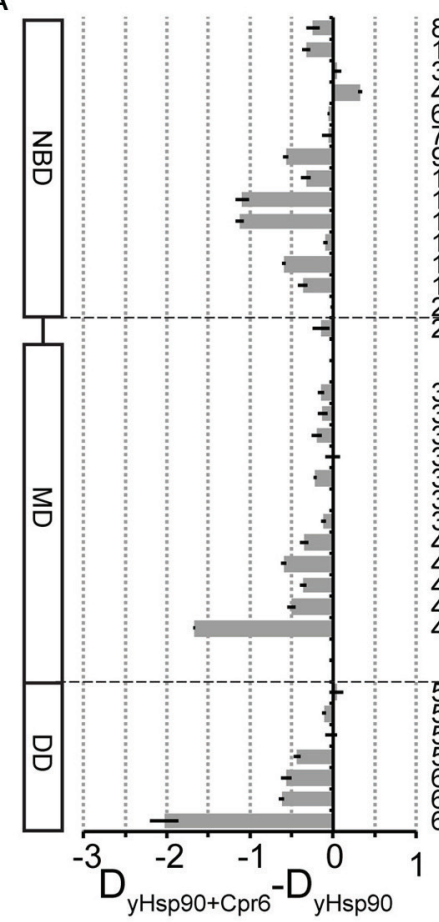

B

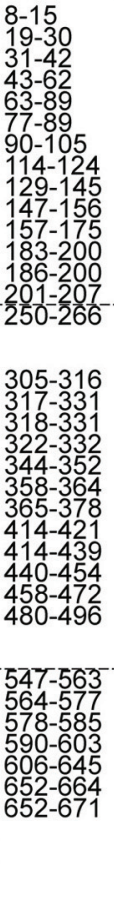

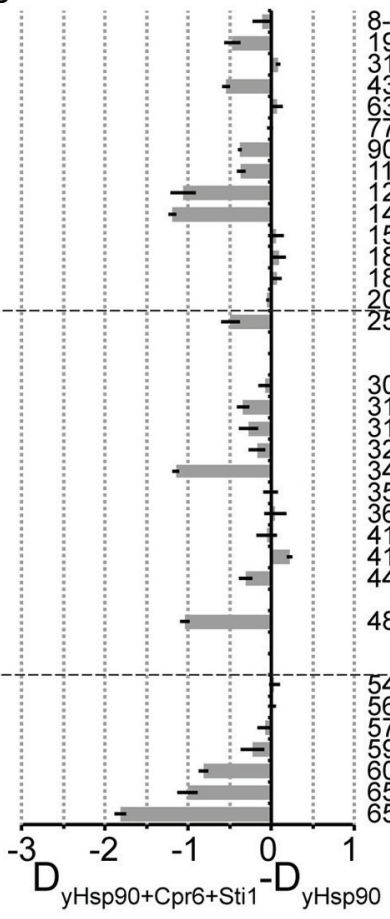

C

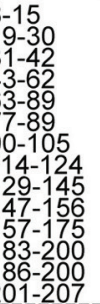

(

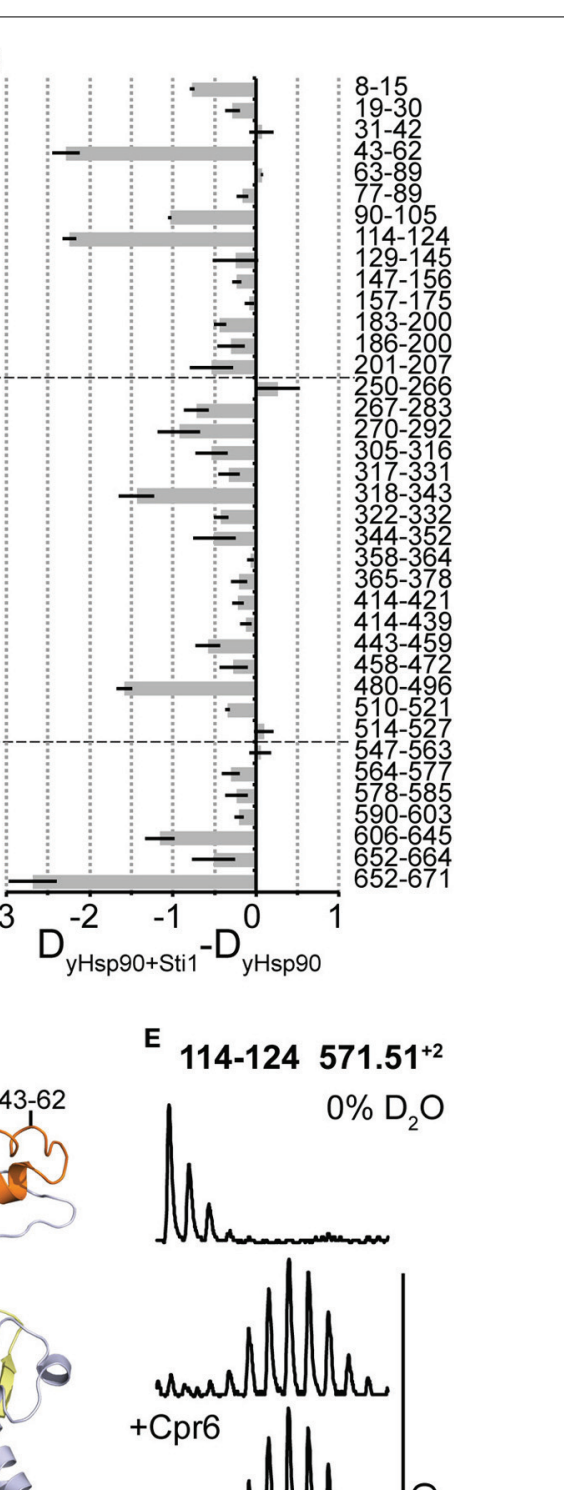

E

114-124 $571.51^{+2}$

D

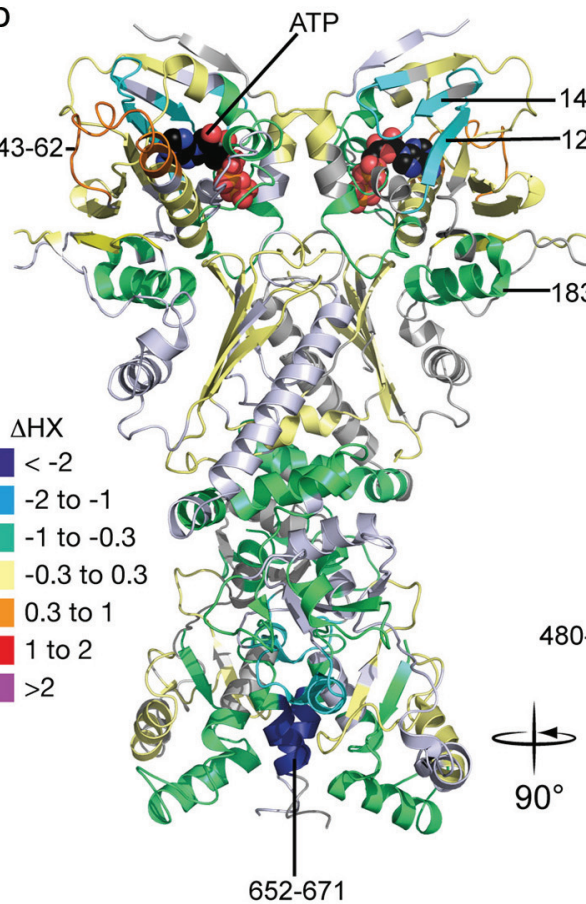

(1,
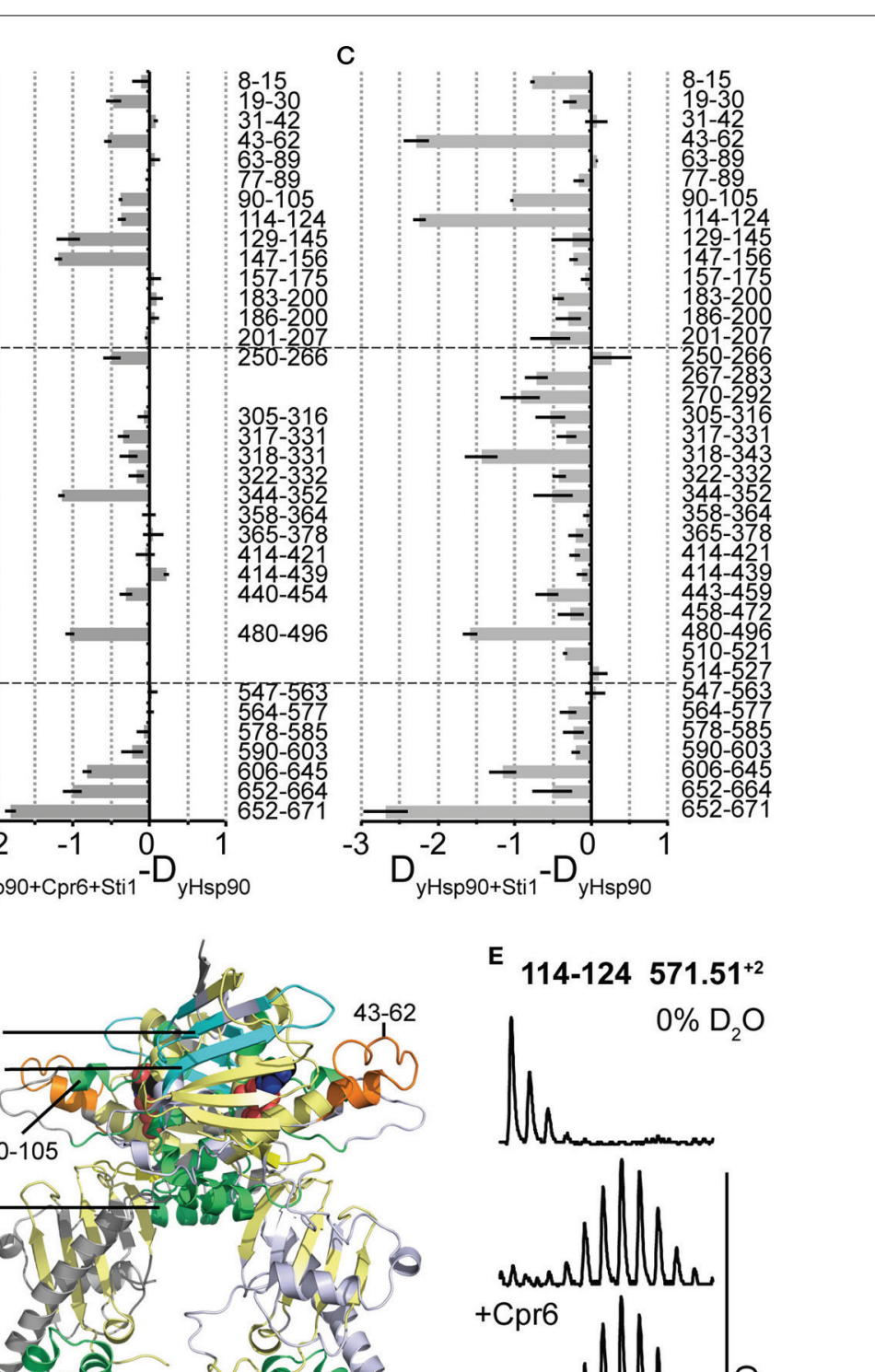

anand|lla

+ Cpr6
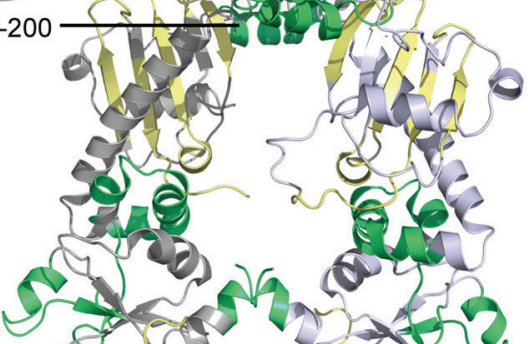

unard Ihe

+ Sti1

$-496-$

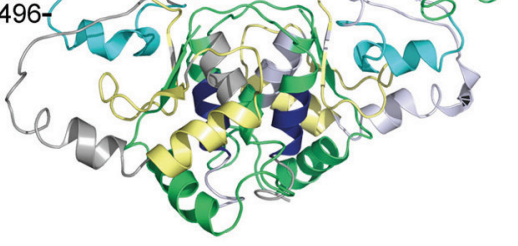

FIGURE 7 | Effects of Cpr6 and Sti1 on the conformational dynamics of yHsp82. (A) Difference plots of deuterons incorporated into yHsp82 in the presence of $\mathrm{Cpr} 6$ minus deuteron incorporation in the absence of $\mathrm{Cpr} 6$ after $30 \mathrm{~s} \mathrm{HX}$. The numbers to the right indicate the corresponding peptic peptides, the domain structure is shown to the left. Error bars represent standard error of three independent experiments. (B) Difference plots of deuterons incorporated into yHsp82 in the presence of Cpr6 and Sti1 minus deuteron incorporation in the absence of Cpr6 and Sti1 after 30 s HX. (C) Difference plots of deuterons incorporated into $\mathrm{yHsp82}$ in the presence of
Sti1 minus deuteron incorporation in the absence of Sti1 after 30s HX (data from Lee et al., 2012). (D) Cartoon representation of yHsp82 (PDB ID 2CG9) colored according to difference of deuteron incorporation into $\mathrm{yHsp} 82$ in the presence of $\mathrm{Cpr6}$ minus deuteron incorporation into $\mathrm{yH}$ sp82 in the absence of Cpr6 as indicated in the color scheme (= heat map for data shown in $\mathbf{A}$ ). (E) Original spectra of the peptic peptide amino acids 114-124 of yHsp82 $(\mathrm{m} / \mathrm{z} 571.51, z=2)$ before $\mathrm{HX}\left(0 \% \mathrm{D}_{2} \mathrm{O}\right)$, or after $30 \mathrm{~s}$ in $\mathrm{D}_{2} \mathrm{O}$ in the absence of co-chaperones, or in the presence of $\mathrm{Cpr} 6$, Sti1 or both $\mathrm{Cpr6}$ and Sti1, from top to bottom as indicated. 


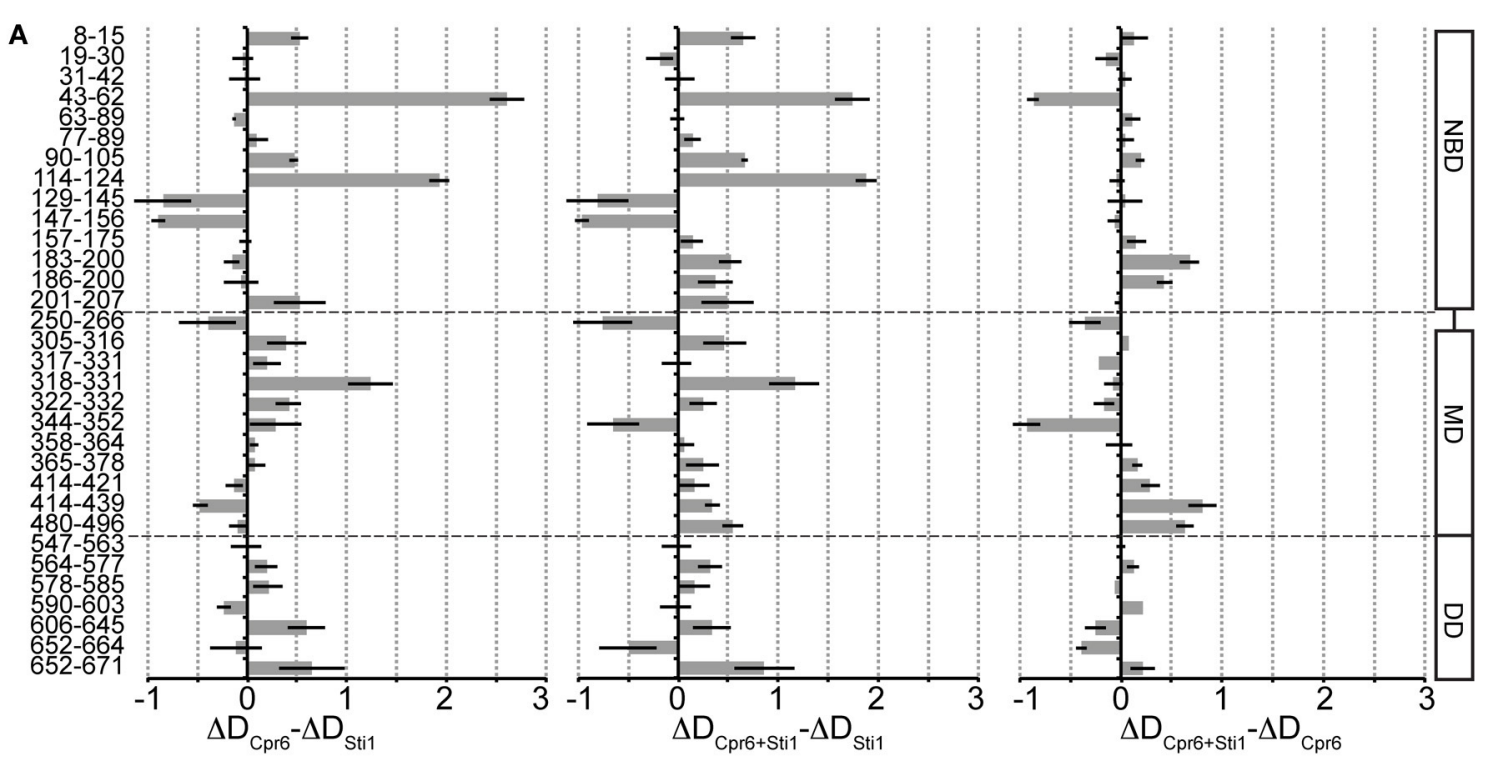

B

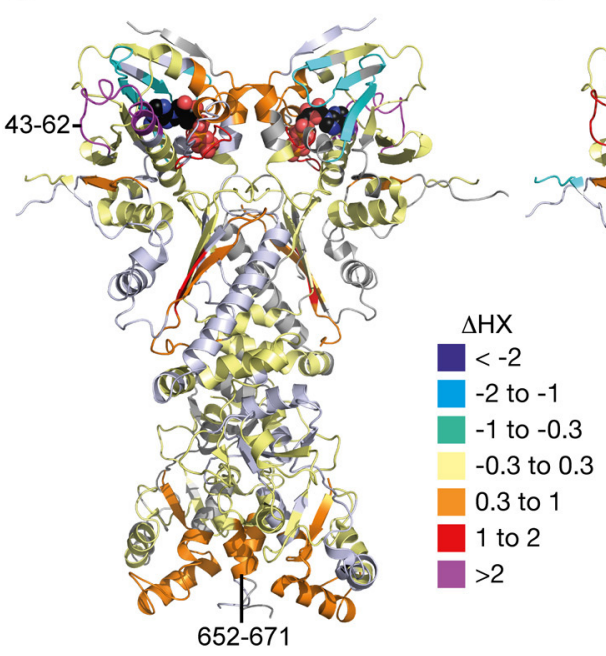

C

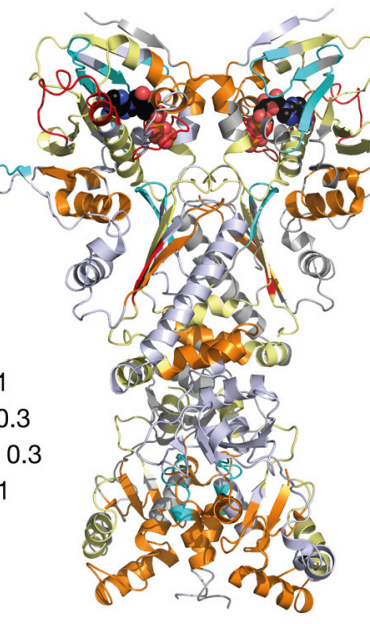

D

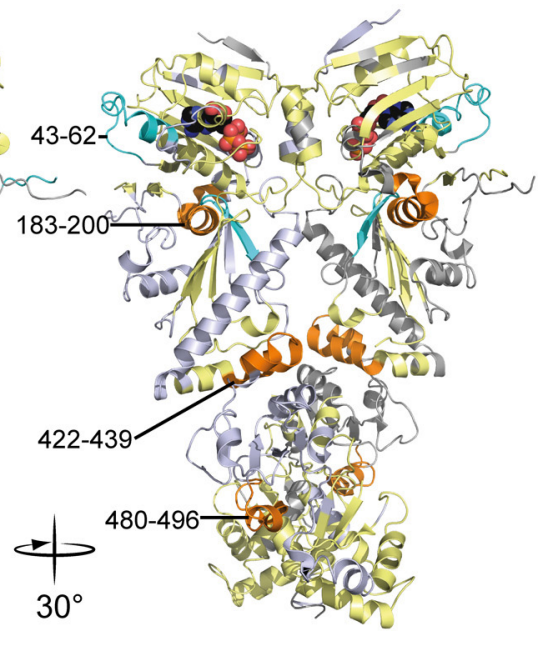

FIGURE 8 | Comparison of Cpr6 and Sti1-induced changes in dynamics of yHsp82. (A) left panel, Difference of Cpr6-induced changes in $\mathrm{HX}$ in $\mathrm{yHsp82}$ as compared to Sti1-induced changes [data (Figure 7A) -data (Figure 7C)]; middle panel, Difference in changes in HX of yHsp82 induced by simultaneous presence of $\mathrm{Cpr} 6$ and Sti1 as compared to presence of Sti1 alone [data (Figure 7B) -data (Figure 7C)]; right panel,
Difference in changes in $\mathrm{HX}$ of $\mathrm{yHsp} 82$ induced by simultaneous presence of Cpr6 and Sti1 as compared to presence of Cpr6 alone [data (Figure 7B) -data (Figure 7A)]. (B-D) Cartoon representations of yHsp82 (PDB ID 2CG9) colored according to the difference plots shown in (A), left panel (B), middle panel (C), and right panel (D) as indicated.

\section{DISCUSSION}

Our analysis of the conformational dynamics of eukaryotic Hsp90 proteins yielded several insights into structure and functioning of Hsp90 proteins. (1) The conformational dynamics of the eukaryotic Hsp90s is much higher than the dynamics of previously studied EcHtpG. (2) As observed for EcHtpG, nucleotideinduced conformational changes are not restricted to the NBD but observed throughout the Hsp90 proteins consistent with published data (Phillips et al., 2007). (3) Eukaryotic Hsp90s are almost as dynamic in the presence of nucleotides as in the apo state consistent with single molecule data (Mickler et al., 2009), and ATP or AMPPNP do not induce a state similar to the ATPbound tensed state of EcHtpG. (4) Co-chaperones stabilize Hsp90 in distinct conformations. (5) Co-chaperones influence each others binding by altering the conformation of Hsp90. (6) Sbal stabilizes Hsp90 in the hydrolysis competent conformation but inhibits ATP hydrolysis by slowing down product release.

Consistent with single molecule measurements on yHsp82 and EcHtpG (Mickler et al., 2009; Ratzke et al., 2012), our data provide strong evidence for the hypothesis that the eukaryotic Hsp90 proteins are probabilistic machines with many different conformational states that are separated only by small energy 

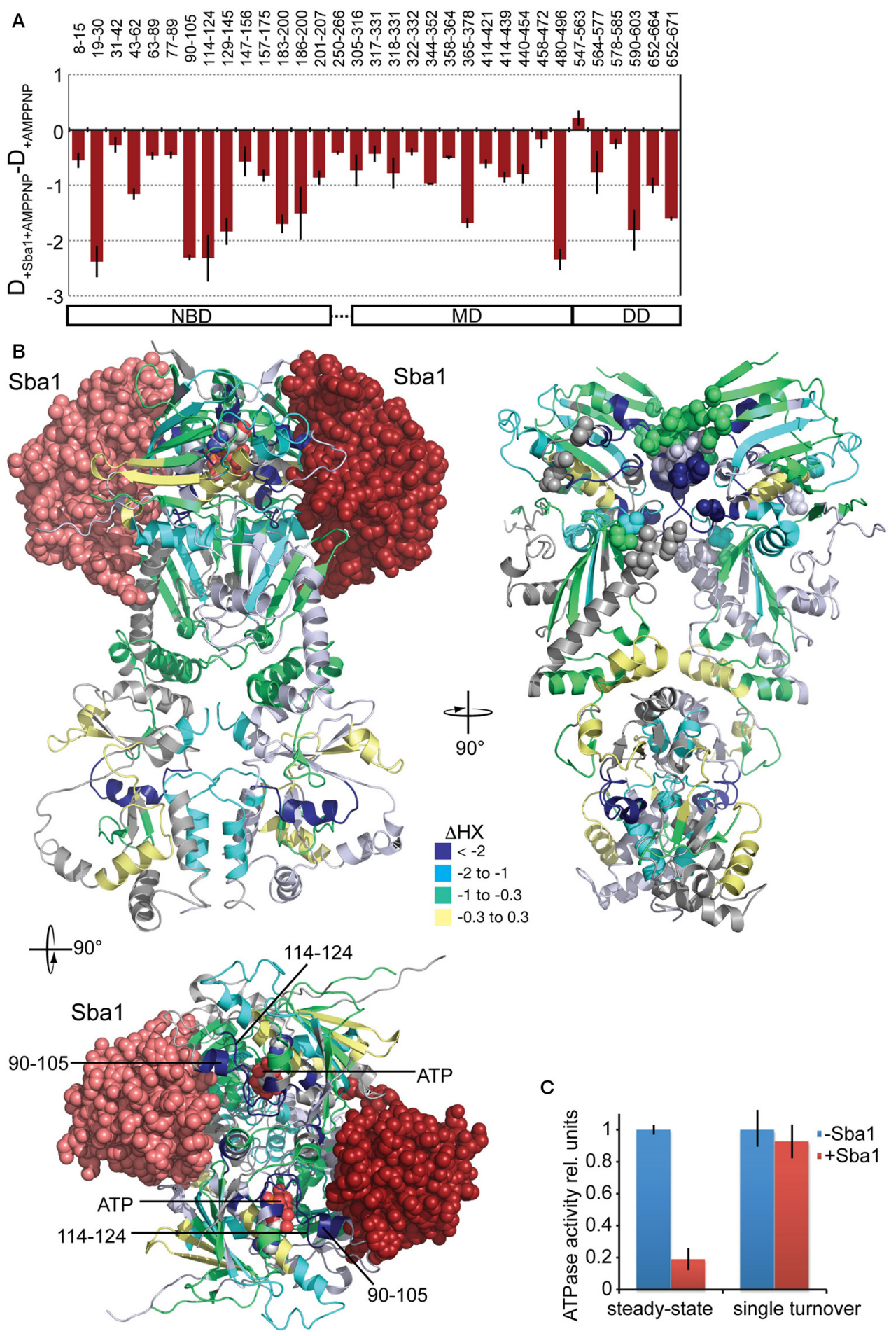

FIGURE 9 | Effects of Sba1 and AMPPNP on the conformational dynamics of yHsp82. (A) Difference plots of deuterons incorporated into yHsp82 in the presence of Sba1 and AMPPNP minus deuteron incorporation in the absence of Sba1 but presence of AMPPNP after $30 \mathrm{~s} \mathrm{HX}$. The numbers on top indicate the corresponding peptic peptides, the domain structure is shown on the bottom. Error bars represent standard error of three independent experiments. (B) Left panel, crystal structure of yHsp82 2 (221-255)-A107N (cartoon representation) in complex with Sba1 (spheres) and AMPPNP. Right panel, same structure rotated as indicated without Sba1 and with Sba1-interacting residues shown as spheres. yHsp82 is colored according to the difference between deuteron incorporation in the presence of Sba1 and deuteron incorporation in the absence of Sba1. Lower panel is a top view of the upper left panel. Segments of the ATP-lid observed in HX experiments are indicated. (C) Sba1 does not inhibit the ATPase activity of yHsp82 under single-turnover conditions. ATPase activity of yHsp82 was determined under steady-state ( $2 \mu \mathrm{M} \mathrm{Hsp82,} 3 \mathrm{mM}$ ATP) and single turnover $(50 \mu \mathrm{M}$ yHsp82, $40 \mu \mathrm{M}$ ATP) conditions in the absence and presence of Sba1 $(60 \mu \mathrm{M})$. 
barriers, while EcHtpG is a deterministic machine with a defined reaction cycle driven by ATP binding and hydrolysis. For eukaryotic Hsp90s, nucleotides alone do not drive a defined reaction cycle, nucleotides and co-chaperones are necessary for an ordered progression through the ATPase cycle.

The higher conformational dynamics in the eukaryotic Hsp90 proteins as compared to the EcHtpG could have several different reasons. The eukaryotic proteins evolved to bind a large number of natively folded proteins, many of which are unrelated in sequence or structure [http://www.picard.ch/downloads/ Hsp90interactors.pdf (Picard, 2006)]. Such a large spectrum of clients may rely on a highly flexible structure to allow Hsp90 to quasi mold into different shapes to provide an optimal interface for client binding. Although it is currently unknown how many and what kind of clients are bound by EcHtpG, it is assumed that these are much lower in number and either not essential themselves or not stringently dependent on EcHtpG, since EcHtpG is not essential under any conditions tested so far and $h t p G$ deletion has a very mild temperature sensitivity phenotype at the upper temperature limit for E. coli growth. Alternatively or in addition, the dynamics of the eukaryotic proteins could also be linked to the interaction with the plethora of co-chaperones. No co-chaperones were identified for EcHtpG so far. While EcHtpG appears to work as a stand-alone machine, the eukaryotic Hsp90s may require the co-chaperones not only to regulate progression through the ATPase cycle but also to stabilize certain parts of the eukaryotic Hsp90 proteins as required for the binding of specific clients.

Consistent with both hypotheses is the fact that the differences in solvent accessibility between EcHtpG and the eukaryotic Hsp90 mainly reside in the $\mathrm{MD}$ and the $\mathrm{DD}$, both of which are implicated in client and co-chaperone binding (Sato et al., 2000; Meyer et al., 2003; Harris et al., 2004). The two co-chaperones tested in this study, Cpr6 and Sba1, both stabilized parts in MD and DD of yHsp82.

HX-MS suggests that the ATPase cycle of all Hsp90s involve similar conformational changes. ATP-induced protection in human and yeast Hsp90s was particularly prominent in the segments, which constitute the hinges of the ATP-lid (residues 99-114 and 126-133 in hHsp90, 90-105 and 114-124 in yHsp82 and 89-98 and 120-127 in EcHtpG). These nucleotide-dependent changes are consistent with structural studies since these segments are found in different conformations in crystal structures of the apo NBD of Hsp90s [PDB ID code 1AH6, 1AH8, 1YES; (Prodromou et al., 1997a,b; Stebbins et al., 1997)] as compared to the full-length structures of yHsp82 and EcHtpG in complex with AMPPNP and Sba1/p23 or ADP [PDB ID codes 2CG9 and 2IOP; (Ali et al., 2006; Shiau et al., 2006)]. They are also consistent with an NMR study of the isolated NBD (Dehner et al., 2003) and of full-length human Hsp90 (Karagöz et al., 2011). However in contrast to the NMR study of full-length Hsp90 (Karagöz et al., 2011), we did not observe any indications of asymmetry in ATP bound Hsp90. The reason for this might be that the observed changes in HX are rather small, making it difficult to detect bimodal distributions, the hallmark of coexisting populations with difference in conformation. Also, the interconversion between the two conformations might be fast on the timescale of our experiments, which would prevent detection by HX-MS.
In addition to the stabilization in the ATP-lid, we found an ATP-induced stabilization in segment 394-415 in hHsp90, a similar protection we observed previously in 318-334 in EcHtpG (Graf et al., 2009). The changes in solvent accessibility in this segment supports the currently favored mechanism of ATP hydrolysis, which assigns a crucial role to arginine 380 in yHsp82 (392 in hHsp90, 336 in EcHtpG) (Meyer et al., 2003, 2004; Ali et al., 2006). A contact between nucleotide and arginine 380 was shown in the crystal structure of full-length yHsp82 in complex with AMPPNP and Sba1/p23 (Ali et al., 2006). Our data now demonstrate that a communication between nucleotide and this arginine-containing loop also occurs in hHsp90 in the presence of ATP and the absence of the Sba1/p23 co-chaperone as previously shown for EcHtpG (Graf et al., 2009). Our data seem to contrast NMR data on full-length human Hsp90 that did not detect perturbations in the chemical shift of the $\delta$-methyl groups of Ile in the MD (Karagöz et al., 2011). The difference between our HX-MS experiments and NMR techniques are the timescale of incubation and data collection, respectively. The full-length crystal structure of yHsp82 also shows an N-terminal dimerization with an exchange of the first $\beta$-strand. In the structure this strand exchange is stabilized by 4 hydrogen bonds. The stabilization observed in the first segments of hHsp90 and yHsp82 was only very small, presumably because any $\mathrm{N}$-terminal dimerization is very transient in the presence of ATP and the absence of stabilizing mutations (Ala107Asn; linker deletion) and co-chaperones like Sba1/p23 like in the crystal structure (Ali et al., 2006). In contrast, in EcHtpG we observed a very prominent protection of almost 8 amide hydrogens in this segment. This observation would be consistent with a stable N-terminal dimerization with strand exchange. The larger number of hydrogen bonds formed in EcHtpG as compared to yHsp82 could be necessary for lack of stabilizing co-chaperones.

Our HX-MS data of yeast and human Hsp90 in the presence of ADP does not provide any evidence for a closed conformation as previously suggested based on electron microscopic data (Shiau et al., 2006; Southworth and Agard, 2008).

Comparison of HX pattern induced by Cpr6 and Stil revealed characteristic differences. Most striking is the segment 43-62, which is strongly protected in the presence of Stil but slightly deprotected in the presence of Cpr6 (Figures 7A,C). This region was suggested to be a direct interaction site between Stil and yHsp82 since it could be cross-linked by a cross-linker positioned in this region (Lee et al., 2012). Cpr6 does not seem to interact with this region. Similarly, the ATP lid (aa 90-105 and aa $114-124$ ) in the NBD and part of the $\beta$-sheet of the MD (aa 318-331) are more protected in the presence of Stil than in the presence of Cpr6. In contrast, parts of the $\beta$-sheet of the NBD (aa 129-156) are more protected in the presence of Cpr6 than in the presence of Stil.

When adding Stil and Cpr6 to yHsp82 at the same time, one of our major concerns was that we may have $y \mathrm{Hsp} 82_{2}$ $\mathrm{Stil}_{2}$ and $\mathrm{yHsp} 82_{2}-\mathrm{Cpr}_{2}$ complexes in addition to the envisioned yHsp82 2 -Sti1-Cpr6 complex. However, the former two complexes would cause a bimodal distribution in the isotope distribution of peptic fragments that differ between the two complexes (e.g. 43-62 and 114-124). Even in a 1:2:1 mixture of yHsp82 $2-\mathrm{Stil}_{2}$ : 
yHsp82 2 -Sti1-Cpr6: yHsp82 2 -Cpr6 2 complexes this should have been visible in a broadening of the isotope distribution. However, this was not observed (see Figure 7E). These data are consistent with published FRET data (Li et al., 2010). The observation that the Stil-induced protection in region 43-64 is strongly reduced in the simultaneous presence of Cpr6 suggests that Cpr6 destabilizes the interaction of Stil with the NBD. The protection in the $\beta$-sheet of the NBD (aa 129-156) in the presence of Stil and Cpr6 are as high as in the presence of Cpr6 alone, suggesting that the conformational dynamics of the NBD are dominated by the interaction with Cpr6. Also in the presence of Stil and Cpr6 the protection of the $\beta$-sheet of the MD (aa 318-331) are reduced as compared to the presence of Stil alone, which might indicate that interaction of Stil with this region is reduced. Surprisingly, we did not observe a bimodal distribution in the regions where the protection pattern differs between Stil and Cpr6 (see Figure 7E). This would be expected, if Stil stably interacts with one protomer and Cpr6 with the other. As no bimodal distributions are observed, both $\mathrm{yHsp} 82$ protomers are identical within the time scale of our experiments ( $30 \mathrm{~s})$. There are two possible explanations for this observation. Both co-chaperones might influence both protomers alike irrespective to which protomer they are bound. Alternatively, the interaction of Stil and Cpr6 with yHsp82 might be dynamic allowing frequent switching of the protomer to which they are bound. The long flexible C-terminal linker that connects the MEEVD motif to the C-terminal dimerization domain and that we previously dubbed fishing line with the MEEVD-motif as hook might allow protomer switching without dissociation from the Hsp90. Both models are consistent with the fact that Stil inhibits the ATPase activity of both protomers even at a stoichiometry of 1:2 Sti1:yHsp82 (Li et al., 2010).

Taken together, our data show that although the primary interaction site for Stil and Cpr6 is the MEEVD motif, both cause stabilization of regions in all three domains of yHsp82 that differs significantly between the two co-chaperones. Our data further suggest that Cpr6 promotes dissociation of Sti1 by destabilizing its contacts to a loop in the NBD and a $\beta$-sheet in the MD.

Sbal binding in the presence of AMPPNP induced a stabilization of yHsp82 in our HX-MS experiments. This was not unexpected since Sbal and its mammalian homolog p23 were shown to stabilize the closed state of Hsp90 (Johnson and Toft, 1995; Johnson et al., 1996; Sullivan et al., 1997; Prodromou et al., 2000). However, with an average of 2 protected amide protons for the 5 most protected peptic fragments the magnitude of stabilization in the N-terminal domain was significantly lower than the stabilization induced by ATP or AMPPNP in EcHtpG [ $>5$ $\mathrm{H}$ for the 5 most protected peptic fragments; Figure 4C; (Graf et al., 2009)]. The most likely explanation for this difference is that the interaction of Sbal/p23 is dynamic and associationdissociation cycles happen during the 30 -s-incubation in $\mathrm{D}_{2} \mathrm{O}$. This is consistent with cross-linking data (C-T. L. and M.P.M, unpublished results) and the fact that for crystallization of fulllength yHsp82 in complex with Sba1/p23 the linker between NBD and MD was shortened by 34 residues (221-255) and, in addition, Ala107 replaced by Asn, an amino acid replacement known to stabilize the closed conformation and to increase the ATPase activity. The protection in the NBD is consistent with a previous NMR study (Karagöz et al., 2011). The protected segments 19-30 and 114-124 include residues corresponding to Ile27, Ile28 and Ile122 in human Hsp90 $\beta$ (19, 20 and 114 yHsp82 numbering) where chemical shifts changes were detected in the presence of $\mathrm{p} 23$.

We also observed protection in the MD and $\mathrm{DD}$, in particular in the dimerization interface. Some of the protected areas in the MD (e.g., 365-378) are consistent with the known crystal structure (Ali et al., 2006), caused either by direct binding of Sbal to $y H s p 82$ or by contacts between MD and NBD in the closed conformation. However, there are also parts of the MD for which protection cannot be explained in this way. In the NMR study some isoleucine residues in the MD also exhibited a shift perturbation (Karagöz et al., 2011). There are two alternative, mutually non-exclusive explanations for these observations. In the crystal structure of yHsp82 in complex with Sba1 the C-terminal 81 residues of Sbal (136-216) were not present since they are unstructured (Weikl et al., 1999). Interaction of these residues with the MD could be responsible for the observed protection in our experiments. Alternatively, stabilization of the N-terminally dimerized conformation of yHsp82 may reduce the dynamics of the $\mathrm{MD}$. The protection observed in the dimerization interface suggests that the slow subunit exchange in the presence of AMPPNP is further reduced upon binding of Sbal.

Several laboratories published earlier that Sba1/p23 inhibits the ATPase activity of Hsp90 (Panaretou et al., 2002; Richter et al., 2004; Siligardi et al., 2004; McLaughlin et al., 2006). Steady-state kinetics suggested a mixed mechanism of inhibition for human p23 (McLaughlin et al., 2006). Interestingly, the degree of inhibition by yeast Sbal seemed to level off at 50\% residual ATPase activity. Based on these data and on earlier observations that Sba1/p23 stabilizes the Hsp90-client complex and that hydrolysis of ATP by Hsp90 leads to client release, it was proposed that Sba1/p23 stabilizes the pre-hydrolysis closed conformation of Hsp90 (Young and Hartl, 2000; Richter et al., 2004; Siligardi et al., 2004; McLaughlin et al., 2006; Prodromou, 2012). The crystal structure of yHsp82 with Sbal shows a hydrolysis competent conformation, but could not distinguish whether Sba1 inhibits ATP hydrolysis by stabilizing the pre-hydrolysis or post-hydrolysis state (Ali et al., 2006). In addition, it was shown recently that the cochaperones Aha1, which stimulates the ATPase activity of Hsp90 by promoting the closed conformation appears to act before Sba1/p23 in the ATPase cycle of Hsp90 (Li et al., 2013). Our HX-MS experiments indicate that Sbal stabilizes the ATP binding lid and most likely the N-terminally dimerized conformation by interacting with both NBDs of the Hsp90 dimer. Our singleturnover experiments demonstrate that Sbal does not inhibit the hydrolysis step itself but the post-hydrolysis product release and most likely the dissociation of the docked NBDs. Whether Sbal enters the Hsp90 complex before hydrolysis or after hydrolysis before the reopening of the closed conformation cannot be decided on the basis of our or previous data. Sba1 might bind with its C-terminal unstructured tail to the Hsp90-bound client waiting for the Aha1-induced N-terminal dimerization of Hsp90 and then bind with high association rates to the docked NBDs. The consequence of this interaction is most likely a stabilization of the Hsp90-client complex as has been proposed earlier (Kosano 
et al., 1998; Morishima et al., 2003; Ali et al., 2006; McLaughlin et al., 2006).

In summary, our data demonstrate that the eukaryotic Hsp90s have increased conformational dynamics as compared to EcHtpG and do not react with large changes in conformational dynamics to binding of nucleotides. In contrast, they need cochaperones to be stabilized in defined conformations necessary for binding and activation of the plethora of their clients. It might be a general tendency in protein evolution within the eukaryotic lineages to lose rigidity and deterministic behavior in order to gain versatility of interaction partners and regulatory potential for cofactors.

\section{MATERIALS AND METHODS REAGENTS}

Fine chemicals were purchased from SIGMA-Aldrich (St. Louis, MO), ATP and ADP were obtained from Roche Applied Science, Mannheim, Germany. Radicicol (RA) was obtained from IRIS Biotech GmbH, Marktredwitz, Germany. Deuterium oxide was purchased from Euriso-top, Gif-sur-Yvette, France. His-tagged Ulp1 was prepared in-house.

\section{PROTEIN EXPRESSION AND PURIFICATION}

Human Hsp90 $\beta$, yeast Hsp82, yeast Hsc82, Cpr6, and Sba1 were cloned into the bacterial expression vector pCA528 encoding an N-terminal His 6 -Smt3 tag (Andréasson et al., 2008). The fusion proteins were overexpressed in the E. coli strain BL21(DE3)Star/pCodonPlus (Invitrogen). The cultures were grown to $\mathrm{OD}_{600}=0.6$ and expression was induced with $0.5 \mathrm{mM}$ IPTG for $5 \mathrm{~h}$ at $30^{\circ} \mathrm{C}$. Cells were lysed by a microfluidizer (Avestin EmulsiFlex-C5) in lysis buffer A $(20 \mathrm{mM}$ $\mathrm{HEPES} / \mathrm{KOH}$ pH 7.5, $100 \mathrm{mM} \mathrm{KCl}, 5 \mathrm{mM} \mathrm{MgCl}, 10 \%$ glycerol, $4 \mathrm{mM} \beta$-mercaptoethanol) and $5 \mathrm{mM}$ PMSF, $1 \mathrm{mM}$ Pepstatin A, $1 \mathrm{mM}$ Leupeptin, $1 \mathrm{mM}$ Aprotinin. The lysate was clarified by centrifugation $(40,000 \mathrm{rpm}$ for $30 \mathrm{~min})$ and incubated with Ni-IDA-matrix (Protino, Macherey-Nagel) for $30 \mathrm{~min}$. After incubation, the matrix was washed with buffer $A$ and bound protein eluted with buffer A containing $250 \mathrm{mM}$ imidazole. The eluted fusion proteins were supplemented with Ulp1 protease, which cleaved the $\mathrm{His}_{6}-\mathrm{Smt} 3$ tag and the mixture was dialyzed overnight against buffer A containing $10 \mathrm{mM} \mathrm{KCl}$. Cleaved recombinant proteins were recovered in the flow-through fractions after a second incubation with Ni-IDA matrix whereas the $\mathrm{N}$-terminal $\mathrm{His}_{6}-\mathrm{Sm} 3 \mathrm{tag}$ and Ulp1 remained on the column. Proteins were further purified by anion exchange chromatography (ReSourceQ, GE Healthcare) with a linear gradient of 0.01-1 M KCl), followed by Superdex 200 size-exclusion chromatography in buffer B (20 mM HEPES 7.6, $300 \mathrm{mM} \mathrm{KCl,} \mathrm{5 \%}$ glycerol, 1mM DTT) and finally dialyzed against storage buffer (40 mM HEPES, pH 7.5, $50 \mathrm{mM} \mathrm{KCl,} 5 \mathrm{mM} \mathrm{MgCl}_{2}, 10 \%$ glycerol, $4 \mathrm{mM} \beta$-mercaptoethanol). The purity and molecular mass was verified by SDS-PAGE and HPLC-electrospray ionization mass spectrometry, confirming the correct primary sequence containing only the $\mathrm{N}$-terminal start-methionine. The purified Hsp90 proteins were checked to be nucleotide-free by anionexchange chromatography (ReSourceQ) and UV detection by $254 \mathrm{~nm}$.

\section{HYDROGEN-EXCHANGE EXPERIMENTS, MASS SPECTROMETRY AND DATA PROCESSING}

Nucleotide-free Hsp90 proteins $(40 \mu \mathrm{M})$ were pre-incubated with a large excess of buffered ATP $(120 \mathrm{mM})$, ADP $(60 \mathrm{mM})$, or AMPPNP $(60 \mathrm{mM})$ for $10 \mathrm{~min}$ at $30^{\circ} \mathrm{C}$ to ensure complete binding. In all cases the ligand occupancy of Hsp90 as calculated from the published dissociation equilibrium constants using the quadratic solution of the binding equilibrium was more than $99 \%$ during pre-incubation and more than $95 \%$ during incubation in $\mathrm{D}_{2} \mathrm{O}$.

HX experiments, mass spectrometry analysis and data processing were performed as described earlier (Rist et al., 2003, 2006; Graf et al., 2009). All experiments were performed at least 3 times independently.

\section{FLUORESCENCE MEASUREMENT}

For the determination of subunit exchange EcHtpG-E58C, yHsp82-E57C and hHsp90ß-E20C,C366A,C412T,C521A,C56 4T,C589A,C590A were labeled with ATTO 488 maleimide and ATTO 550 maleimide (ATTO-TEC GmbH). Corresponding ATTO 488 and ATTO 550 labeled proteins were combined (400 $\mathrm{nM}$ each) and rapidly mixed 1:1 with unlabeled protein $(20 \mu \mathrm{M})$ in a stopped-flow device (Applied Photophysics) with $480 \mathrm{~nm}$ excitation and $590 \mathrm{~nm}$ cut-off filter.

\section{ATPase ASSAYS}

Steady-state and single-turnover ATPase assays were determined as described earlier (Ali et al., 1993; Graf et al., 2009) except that $25 \mathrm{mM}$ HEPES-KOH pH 7.5, $20 \mathrm{mM} \mathrm{KCl}, 5 \mathrm{mM} \mathrm{MgCl}_{2}$ was used as buffer.

\section{AUTHOR CONTRIBUTIONS}

Christian Graf cloned genes, constructed and purified wild type and mutant proteins; performed experiments and analyzed data for Figures 1-4; prepared Figures 1, 2A; contributed to manuscript writing. Chung-Tien Lee constructed and purified wild type and mutant proteins; performed experiments and analyzed data for Figures 2C, 3, 4B,C, 5, 6C, 8; contributed to manuscript writing. L. Eva Meier-Andrejszki purified Cpr6 and performed experiments and analyzed data for Figures 7A,B; Minh T. N. Nguyen constructed mutant variants and performed ATPase assays (Figure 9C). Matthias P. Mayer designed and supervised experiments, analyzed data, prepared figures and wrote the manuscript.

\section{ACKNOWLEDGMENTS}

We thank Z. Zhang for providing the program HXPep, M. Boysen for providing hHsp90ß-E20C,C366A,C412T,C521A,C5 64T,C589A,C590A, and T. Ruppert for his support in the mass spectrometry facility. This work was supported by the Deutsche Forschungsgemeinschaft (SFB638).

\section{REFERENCES}

Ali, J. A., Jackson, A. P., Howells, A. J., and Maxwell, A. (1993). The 43-kilodalton N-terminal fragment of the DNA gyrase B protein hydrolyzes ATP and binds coumarin drugs. Biochemistry 32, 2717-2724. doi: 10.1021/bi00061a033

Ali, M. M. U., Roe, S. M., Vaughan, C. K., Meyer, P., Panaretou, B., Piper, P. W., et al. (2006). Crystal structure of an Hsp90-nucleotide-p23/Sbal closed chaperone complex. Nature 440, 1013-1017. doi: 10.1038/nature04716 
Andréasson, C., Fiaux, J., Rampelt, H., Mayer, M. P., and Bukau, B. (2008). Hsp110 is a nucleotide-activated exchange factor for Hsp70. J. Biol. Chem. 283, 8877-8884. doi: 10.1074/jbc.M710063200

Arnold, K., Bordoli, L., Kopp, J., and Schwede, T. (2006). The SWISS-MODEL workspace: a web-based environment for protein structure homology modelling. Bioinformatics 22, 195-201. doi: 10.1093/bioinformatics/bti770

Dehner, A., Furrer, J., Richter, K., Schuster, I., Buchner, J., and Kessler, H. (2003). NMR chemical shift perturbation study of the N-terminal domain of Hsp90 upon binding of ADP, AMP-PNP, geldanamycin, and radicicol. Chembiochem 4, 870-877. doi: 10.1002/cbic.200300658

DeZwaan, D. C., and Freeman, B. C. (2008). HSP90: the Rosetta stone for cellular protein dynamics? Cell Cycle 7, 1006-1012. doi: 10.4161/cc.7.8.5723

Dollins, D. E., Warren, J. J., Immormino, R. M., and Gewirth, D. T. (2007). Structures of GRP94-nucleotide complexes reveal mechanistic differences between the hsp90 chaperones. Mol. Cell 28, 41-56. doi: 10.1016/j.molcel.2007.08.024

Fang, Y., Fliss, A. E., Rao, J., and Caplan, A. J. (1998). SBA1 encodes a yeast hsp90 cochaperone that is homologous to vertebrate p23 proteins. Mol. Cell. Biol. 18 , 3727-3734.

Ghaemmaghami, S., Huh, W.-K., Bower, K., Howson, R. W., Belle, A., Dephoure, N., et al. (2003). Global analysis of protein expression in yeast. Nature 425 , 737-741. doi: 10.1038/nature02046

Graf, C., Stankiewicz, M., Kramer, G., and Mayer, M. P. (2009). Spatially and kinetically resolved changes in the conformational dynamics of the Hsp90 chaperone machine. EMBO J. 28, 602-613. doi: 10.1038/emboj.2008.306

Guex, N., Peitsch, M. C., and Schwede, T. (2009). Automated comparative protein structure modeling with SWISS-MODEL and Swiss-PdbViewer: a historical perspective. Electrophoresis 30(Suppl. 1), S162-S173. doi: 10.1002/elps. 200900140

Harris, S. F., Shiau, A. K., and Agard, D. A. (2004). The crystal structure of the carboxy-terminal dimerization domain of htpG, the Escherichia coli Hsp90, reveals a potential substrate binding site. Structure 12, 1087-1097. doi: 10.1016/j.str.2004.03.020

Hessling, M., Richter, K., and Buchner, J. (2009). Dissection of the ATP-induced conformational cycle of the molecular chaperone Hsp90. Nat. Struct. Mol. Biol. 16, 287-293. doi: 10.1038/nsmb.1565

Hoofnagle, A. N., Resing, K. A., and Ahn, N. G. (2003). Protein analysis by hydrogen exchange mass spectrometry. Annu. Rev. Biophys. Biomol. Struct. 32, 1-25. doi: 10.1146/annurev.biophys.32.110601.142417

Johnson, J., Corbisier, R., Stensgard, B., and Toft, D. (1996). The involvement of p23, hsp90, and immunophilins in the assembly of progesterone receptor complexes. J. Steroid Biochem. Mol. Biol. 56, 31-37. doi: 10.1016/09600760(95)00221-9

Johnson, J. L., and Toft, D. O. (1995). Binding of p23 and hsp90 during assembly with the progesterone receptor. Mol. Endocrinol. 9, 670-678.

Karagöz, G. E., Duarte, A. M. S., Ippel, H., Uetrecht, C., Sinnige, T., van Rosmalen, M., et al. (2011). N-terminal domain of human Hsp90 triggers binding to the cochaperone p23. Proc. Natl. Acad. Sci. U.S.A. 108, 580-585. doi: $10.1073 /$ pnas. 1011867108

Kiefer, F., Arnold, K., Künzli, M., Bordoli, L., and Schwede, T. (2009). The SWISS-MODEL Repository and associated resources. Nucleic Acids Res. 37, D387-D392. doi: 10.1093/nar/gkn750

Kosano, H., Stensgard, B., Charlesworth, M. C., McMahon, N., and Toft, D. (1998). The assembly of progesterone receptor-hsp90 complexes using purified proteins. J. Biol. Chem. 273, 32973-32979. doi: 10.1074/jbc.273.49.32973

Krukenberg, K. A., Southworth, D. R., Street, T. O., and Agard, D. A. (2009). pH-dependent conformational changes in bacterial Hsp90 reveal a Grp94like conformation at $\mathrm{pH} 6$ that is highly active in suppression of citrate synthase aggregation. J. Mol. Biol. 390, 278-291. doi: 10.1016/j.jmb.2009. 04.080

Lee, C.-T., Graf, C., Mayer, F. J., Richter, S. M., and Mayer, M. P. (2012). Dynamics of the regulation of Hsp90 by the co-chaperone Stil. EMBO J. 31, 1518-1528. doi: 10.1038/emboj.2012.37

Li, J., Richter, K., and Buchner, J. (2010). Mixed Hsp90-cochaperone complexes are important for the progression of the reaction cycle. Nat. Struct. Mol. Biol. 18, 61-66. doi: 10.1038/nsmb. 1965

Li, J., Richter, K., Reinstein, J., and Buchner, J. (2013). Integration of the accelerator Ahal in the Hsp90 co-chaperone cycle. Nat. Struct. Mol. Biol. 20, 326-331. doi: $10.1038 / \mathrm{nsmb} .2502$
McLaughlin, S. H., Sobott, F., Yao, Z.-P., Zhang, W., Nielsen, P. R., Grossmann, J. G., et al. (2006). The co-chaperone p23 arrests the Hsp90 ATPase cycle to trap client proteins. J. Mol. Biol. 356, 746-758. doi: 10.1016/j.jmb. 2005.11.085

Meyer, P., Prodromou, C., Hu, B., Vaughan, C., Roe, S. M., Panaretou, B., et al. (2003). Structural and functional analysis of the middle segment of hsp90: implications for ATP hydrolysis and client protein and cochaperone interactions. Mol. Cell 11, 647-658. doi: 10.1016/S1097-2765(03)00065-0

Meyer, P., Prodromou, C., Liao, C., Hu, B., Roe, S. M., Vaughan, C. K., et al. (2004). Structural basis for recruitment of the ATPase activator Ahal to the Hsp90 chaperone machinery. EMBO J. 23, 1402-1410. doi: 10.1038/sj.emboj. 7600141

Mickler, M., Hessling, M., Ratzke, C., Buchner, J., and Hugel, T. (2009). The large conformational changes of Hsp90 are only weakly coupled to ATP hydrolysis. Nat. Struct. Mol. Biol. 16, 281-286. doi: 10.1038/nsmb.1557

Morishima, Y., Kanelakis, K. C., Murphy, P. J. M., Lowe, E. R., Jenkins, G. J., Osawa, Y., et al. (2003). The hsp90 cochaperone p23 is the limiting component of the multiprotein hsp90/hsp70-based chaperone system in vivo where it acts to stabilize the client protein: hsp90 complex. J. Biol. Chem. 278, 48754-48763. doi: 10.1074/jbc.M309814200

Panaretou, B., Siligardi, G., Meyer, P., Maloney, A., Sullivan, J. K., Singh, S., et al. (2002). Activation of the ATPase activity of hsp90 by the stressregulated cochaperone aha1. Mol. Cell 10, 1307-1318. doi: 10.1016/S1097 2765(02)00785-2

Pearl, L. H., and Prodromou, C. (2000). Structure and in vivo function of Hsp90. Curr. Opin. Struct. Biol. 10, 46-51. doi: 10.1016/S0959-440X(99) 00047-0

Pearl, L. H., and Prodromou, C. (2006). Structure and mechanism of the Hsp90 molecular chaperone machinery. Annu. Rev. Biochem. 75, 271-294. doi: 10.1146/annurev.biochem.75.103004.142738

Phillips, J. J., Yao, Z.-P., Zhang, W., McLaughlin, S., Laue, E. D., Robinson, C. V., et al. (2007). Conformational dynamics of the molecular chaperone Hsp90 in complexes with a co-chaperone and anticancer drugs. J. Mol. Biol. 372, 1189-1203. doi: 10.1016/j.jmb.2007.04.059

Picard, D. (2006). Chaperoning steroid hormone action. Trends Endocrinol. Metab. 17, 229-235. doi: 10.1016/j.tem.2006.06.003

Pratt, W. B., and Toft, D. O. (1997). Steroid receptor interactions with heat shock protein and immunophilin chaperones. Endocr. Rev. 18, 306-360.

Prodromou, C. (2012). The "active life" of Hsp90 complexes. Biochim. Biophys. Acta 1823, 614-623. doi: 10.1016/j.bbamcr.2011.07.020

Prodromou, C., Panaretou, B., Chohan, S., Siligardi, G., O’Brien, R., Ladbury, J. E., et al. (2000). The ATPase cycle of Hsp90 drives a molecular "clamp" via transient dimerization of the N-terminal domains. EMBO J. 19, 4383-4392. doi: 10.1093/emboj/19.16.4383

Prodromou, C., Roe, S. M., O’Brien, R., Ladbury, J. E., Piper, P. W., and Pearl, L. H. (1997a). Identification and structural characterization of the ATP/ADP-binding site in the Hsp90 molecular chaperone. Cell 90, 65-75.

Prodromou, C., Roe, S. M., Piper, P. W., and Pearl, L. H. (1997b). A molecular clamp in the crystal structure of the N-terminal domain of the yeast Hsp90 chaperone. Nat. Struct Biol. 4, 477-482. doi: 10.1038/nsb0697-477

Ratzke, C., Mickler, M., Hellenkamp, B., Buchner, J., and Hugel, T. (2010). Dynamics of heat shock protein $90 \mathrm{C}$-terminal dimerization is an important part of its conformational cycle. Proc. Natl. Acad. Sci. U.S.A. 107, 16101-16106. doi: 10.1073/pnas.1000916107

Ratzke, C., Nguyen, M. N. T., Mayer, M. P., and Hugel, T. (2012). From a ratchet mechanism to random fluctuations evolution of Hsp90's mechanochemical cycle. J. Mol. Biol. 423, 462-471. doi: 10.1016/j.jmb.2012.07.026

Richter, K., Muschler, P., Hainzl, O., and Buchner, J. (2001). Coordinated ATP hydrolysis by the Hsp90 dimer. J. Biol. Chem. 276, 33689-33696. doi: 10.1074/jbc.M103832200

Richter, K., Walter, S., and Buchner, J. (2004). The Co-chaperone Sbal connects the ATPase reaction of Hsp90 to the progression of the chaperone cycle. J. Mol. Biol. 342, 1403-1413. doi: 10.1016/j.jmb.2004.07.064

Rist, W., Graf, C., Bukau, B., and Mayer, M. P. (2006). Amide hydrogen exchange reveals conformational changes in hsp70 chaperones important for allosteric regulation. J. Biol. Chem. 281, 16493-16501. doi: 10.1074/jbc. M600847200

Rist, W., Jørgensen, T. J. D., Roepstorff, P., Bukau, B., and Mayer, M. P. (2003). Mapping temperature-induced conformational changes in the Escherichia coli 
heat shock transcription factor sigma 32 by amide hydrogen exchange. J. Biol. Chem. 278, 51415-51421. doi: 10.1074/jbc.M307160200

Sato, S., Fujita, N., and Tsuruo, T. (2000). Modulation of Akt kinase activity by binding to Hsp90. Proc. Natl. Acad. Sci. U.S.A. 97, 10832-10837. doi: 10.1073/pnas. 170276797

Shiau, A. K., Harris, S. F., Southworth, D. R., and Agard, D. A. (2006). Structural Analysis of E. coli hsp 90 reveals dramatic nucleotide-dependent conformational rearrangements. Cell 127, 329-340. doi: 10.1016/j.cell.2006.09.027

Siligardi, G., Hu, B., Panaretou, B., Piper, P. W., Pearl, L. H., and Prodromou, C. (2004). Co-chaperone regulation of conformational switching in the Hsp90 ATPase cycle. J. Biol. Chem. 279, 51989-51998. doi: 10.1074/jbc. M410562200

Southworth, D. R., and Agard, D. A. (2008). Species-dependent ensembles of conserved conformational states define the Hsp90 chaperone ATPase cycle. Mol. Cell 32, 631-640. doi: 10.1016/j.molcel.2008.10.024

Sreedhar, A. S., Kalmár, E., Csermely, P., and Shen, Y.-F. (2004). Hsp90 isoforms: functions, expression and clinical importance. FEBS Lett. 562, 11-15. doi: 10.1016/S0014-5793(04)00229-7

Stebbins, C. E., Russo, A. A., Schneider, C., Rosen, N., Hartl, F. U., and Pavletich, N. P. (1997). Crystal structure of an Hsp90-geldanamycin complex: targeting of a protein chaperone by an antitumor agent. Cell 89, 239-250. doi: 10.1016/S00928674(00)80203-2

Sullivan, W., Stensgard, B., Caucutt, G., Bartha, B., McMahon, N., Alnemri, E. S., et al. (1997). Nucleotides and two functional states of hsp90. J. Biol. Chem. 272, 8007-8012. doi: 10.1074/jbc.272.12.8007

Wales, T. E., and Engen, J. R. (2006). Hydrogen exchange mass spectrometry for the analysis of protein dynamics. Mass Spectrom. Rev. 25, 158-170. doi: 10.1002/mas.20064
Wegele, H., Müller, L., and Buchner, J. (2004). Hsp70 and Hsp90-a relay team for protein folding. Rev. Physiol. Biochem. Pharmacol. 151, 1-44. doi: 10.1007/s10254-003-0021-1

Weikl, T., Abelmann, K., and Buchner, J. (1999). An unstructured C-terminal region of the Hsp90 co-chaperone $\mathrm{p} 23$ is important for its chaperone function. J. Mol. Biol. 293, 685-691. doi: 10.1006/jmbi.1999.3172

Young, J. C., and Hartl, F. U. (2000). Polypeptide release by Hsp90 involves ATP hydrolysis and is enhanced by the co-chaperone p23. EMBO J. 19, 5930-5940. doi: $10.1093 / \mathrm{emboj} / 19.21 .5930$

Young, J. C., Moarefi, I., and Hartl, F. U. (2001). Hsp90: a specialized but essential protein-folding tool. J. Cell Biol. 154, 267-273. doi: 10.1083/jcb.200104079

Conflict of Interest Statement: The authors declare that the research was conducted in the absence of any commercial or financial relationships that could be construed as a potential conflict of interest.

Received: 03 April 2014; accepted: 21 May 2014; published online: 10 June 2014. Citation: Graf C, Lee C-T, Eva Meier-Andrejszki L, Nguyen MTN and Mayer MP (2014) Differences in conformational dynamics within the Hsp90 chaperone family reveal mechanistic insights. Front. Mol. Biosci. 1:4. doi: 10.3389/fmolb.2014.00004 This article was submitted to Protein Folding, Misfolding and Degradation, a section of the journal Frontiers in Molecular Biosciences.

Copyright $\odot 2014$ Graf, Lee, Eva Meier-Andrejszki, Nguyen and Mayer. This is an open-access article distributed under the terms of the Creative Commons Attribution License (CC BY). The use, distribution or reproduction in other forums is permitted, provided the original author(s) or licensor are credited and that the original publication in this journal is cited, in accordance with accepted academic practice. No use, distribution or reproduction is permitted which does not comply with these terms. 